\title{
White dwarf and subdwarf stars in the Sloan Digital Sky Survey Data Release 14
}

\section{S. O. Kepler ${ }^{\odot},{ }^{1 \star}$ Ingrid Pelisoli ${ }^{\odot},{ }^{1,2}$ Detlev Koester, ${ }^{3}$ Nicole Reindl, ${ }^{4}$ Stephan Geier, ${ }^{2}$ Alejandra D. Romero ${ }^{\odot},{ }^{1}$ Gustavo Ourique, ${ }^{1}$ Cristiane de Paula Oliveira ${ }^{1}$ and Larissa} A. Amaral ${ }^{1}$

${ }^{1}$ Instituto de Física, Universidade Federal do Rio Grande do Sul, 91501-900 Porto-Alegre, RS, Brazil

${ }_{2}^{2}$ Institut für Physik und Astronomie, Universitätsstandort Golm, Karl-Liebknecht-Str 24/25, D-14467 Potsdam, Germany

${ }^{3}$ Institut für Theoretische Physik und Astrophysik, Universität Kiel, D-24098 Kiel, Germany

${ }^{4}$ Department of Physics and Astronomy, University of Leicester, University Road, Leicester LE1 7RH, UK

Accepted 2019 April 1. Received 2019 February 14; in original form 2018 October 22

\begin{abstract}
White dwarfs carry information on the structure and evolution of the Galaxy, especially through their luminosity function and initial-to-final mass relation. Very cool white dwarfs provide insight into the early ages of each population. Examining the spectra of all stars with $3 \sigma$ proper motion in the Sloan Digital Sky Survey Data Release 14, we report the classification for 20088 spectroscopically confirmed white dwarfs, plus 415 hot subdwarfs, and 311 cataclysmic variables. We obtain $T_{\text {eff }}, \log g$, and mass for hydrogen atmosphere white dwarf stars (DAs), warm helium atmosphere white dwarfs (DBs), hot subdwarfs (sdBs and sdOs), and estimate photometric $T_{\text {eff }}$ for white dwarf stars with continuum spectra (DCs). We find 15793 sdAs and $447 \mathrm{dCs}$ between the white dwarf cooling sequence and the main sequence, especially below $T_{\text {eff }} \simeq 10000 \mathrm{~K}$; most are likely low-mass metal-poor main-sequence stars, but some could be the result of interacting binary evolution.
\end{abstract}

Key words: catalogues - subdwarfs - white dwarfs; stars: fundamental parameters; cataclysmic variables;.

\section{INTRODUCTION}

White dwarf stars are the end state for all stars formed with initial masses below around 7-11.8 $\mathrm{M}_{\odot}$, depending on metallicity (e.g. Ibeling \& Heger 2013; Doherty et al. 2015; Woosley \& Heger 2015; Lauffer, Romero \& Kepler 2018), which translates to more than 97 percent of all stars. Therefore the properties of the white dwarf population reflect the result of the initial mass function, the star formation rate, and the initial-to-final mass relation, for different metallicities. White dwarf stars are also possible outcomes of the evolution of multiple systems, with 25-30 per cent of white dwarfs estimated to be the result of mergers (Toonen et al. 2017). White dwarfs with masses lower than $0.3-0.45 \mathrm{M}_{\odot}$ are generally explained as outcomes of close binary evolution (Kilic, Stanek \& Pinsonneault 2007), given that the single progenitors of such low-mass white dwarfs have main-sequence lifetimes exceeding the age of the Universe. The formation mechanism of the so-called extremely low mass white dwarfs (ELMs) - those with masses below $\simeq 0.2-0.3 \mathrm{M}_{\odot}$ (e.g. Sun \& Arras 2018; Calcaferro, Althaus \& Córsico 2018, and references therein) - is similar to that proposed to explain composite

^E-mail: kepler@if.ufrgs.br hot subdwarf stars (e.g. Heber 2016): the outer envelope is lost after a common envelope or a stable Roche lobe overflow phase, leaving the stellar core exposed (e.g. Li et al. 2019). Hot subdwarfs result when the envelope is lost after He-burning is triggered in the core - hence they lie above the zero-age horizontal branch (ZAHB), whereas an ELM will result if the mass is lost when the core He is in a degenerate state, but He fusion has not been triggered. ELMs show similar log $g$ to subdwarfs, but generally lower temperature $\left(T_{\text {eff }} \lesssim 20000 \mathrm{~K}\right)$.

White dwarfs do not present ongoing core nuclear burning, but residual shell burning may occur depending on the thickness of the hydrogen layer. ELMs are believed to show residual burning before reaching the final white dwarf cooling track (Córsico et al. 2012; Istrate et al. 2016). This happens in the pre-ELM phase (Maxted et al. 2014a,b), which can cause them to show luminosities comparable to main-sequence and even horizontal branch stars (e.g. Pietrzyński et al. 2012).

Because the time-scales for gravitational settling are of the order of a few million years or smaller, the atmospheric composition of white dwarf stars is generally simple, with around 80 per cent showing solely $\mathrm{H}$ lines (spectral class DA). The remaining are dominated by He lines, when the atmospheric temperature is sufficient to excite 
the He atoms. The spectral class is DB if only He I lines are present, and DO if He II lines are visible (typically $T_{\text {eff }} \gtrsim 40000 \mathrm{~K}$ ). Very cool white dwarfs ( $T_{\text {eff }} \lesssim 5000 \mathrm{~K}$ for $\mathrm{H}$ atmosphere, $T_{\text {eff }} \lesssim 11000 \mathrm{~K}$ for $\mathrm{He}$ atmosphere) show featureless spectra and are classified as DCs. A substantial fraction (20-50 percent, Zuckerman et al. 2003; Koester, Gänsicke \& Farihi 2014) of white dwarfs show contamination by metals, which can only be explained by ongoing accretion, except for very hot objects $\left(T_{\text {eff }} \gtrsim 50000 \mathrm{~K}\right)$, where radiative levitation can still play a significant role (e.g. Barstow et al. 2014); a $\mathrm{Z}$ is added to the spectral classification to flag metal pollution. In rare cases, for stars classified as DQs, carbon may be dragged to the surface by convection (e.g. Koester, Weidemann \& Zeidler 1982). Cool DQs show spectra similar to dwarf carbon (dC) stars, which are themselves believed to be one outcome binary evolution (Whitehouse et al. 2018).

In this paper we extend the work of Kleinman et al. (2013) and Kepler et al. (2015, 2016), continuing the search for new spectroscopically confirmed white dwarf and subdwarf stars in the data release 14 of the Sloan Digital Sky Survey (SDSS DR14; Abolfathi et al. 2018). Spectroscopy allows precise determinations of $T_{\text {eff }}, \log g$, and abundances, serving as a valuable resource for studying stellar formation and evolution in the Milky Way (e.g. Winget et al. 1987; Bergeron, Saffer \& Liebert 1992; Liebert, Bergeron \& Holberg 2005; Tremblay et al. 2014). As a by-product, we also identify cataclysmic variables (CVs) - white dwarfs with ongoing mass exchange from a companion, and presenting emission lines, generally of hydrogen and/or helium - and dC stars, due to their similarity with carbon-rich white dwarfs. These dC stars (Roulston et al. 2018), as well as hot subdwarfs and ELMs, hold potential to shed light on the poorly understood process of close binary evolution.

\section{DATA ANALYSIS}

\subsection{Identification of the candidates}

We started with the 4851200 optical spectra in the SDSS DR14. We selected the 259537 spectra of stars with $3 \sigma$ proper motion larger than $20 \mathrm{mas} \mathrm{yr}^{-1}$, as well as all 68836 newly observed spectra of stars with colours within the Kleinman et al. (2013) selected white dwarf colour range, and all 225471 spectra classified by the SDSS spectral pipeline as WHITE_DWARF, A, B, OB, or O stars, or CV. In addition, we performed an automated search for similar spectra as described in Kepler et al. $(2015,2016)$ on all the 4851200 optical spectra, selecting further $\approx 4000$ spectra. We examined these selected spectra by eye $(\approx 500000$ spectra, given the overlap between the different selections) to identify broadline spectra characteristic of white dwarfs, hot subdwarfs, and $\mathrm{dCs}$, resulting in our identification of 34321 high-signal-to-noise $\left(\mathrm{S} / \mathrm{N}_{g}\right)$ spectra containing white dwarf, subdwarf, CVs, and $\mathrm{dCs}$ stars. $\mathrm{S} / \mathrm{N}_{g}$ is the $\mathrm{S} / \mathrm{N}$ parameter in the $g$ band in the SDSS spectra reduction pipeline. Our visual inspection showed that most objects in the SDSS catalogue with proper motion smaller than $30{\text { mas } \mathrm{yr}^{-1}}^{-1}$ and magnitude $g>20$ are in fact galaxies, from their composite spectrum, high redshifted lines, or broad emission lines. We also inspected 1449 additional spectra for Gaia DR2 stars in the colourmagnitude white dwarf region $\left[M_{\mathrm{GG}}>3.333 \times\left(\mathrm{G}_{\mathrm{BP}}-\mathrm{G}_{\mathrm{RP}}\right)+\right.$ 8.333], not included in our previous selection. This white dwarf region was selected using the photometric conditions in Kilic et al. (2018), but with parallax/error $>4$, flux/error $>3$, as we are looking for stars with spectra and SDSS photometry, matching to 3 arcsec in the SDSS coordinates.
In previous SDSS white dwarf catalogues, we had not employed a proper motion criterion for selection, obtaining an $\mathrm{S} / \mathrm{N}$ limited sample, determined by a colour-magnitude selection. The main reason we expanded our selection to include low-S/N spectra from high proper motion objects is that our previous colour selection excluded the low-temperature white dwarfs $\left(T_{\text {eff }}<8000 \mathrm{~K}\right)$, because their SDSS colours are similar to the more numerous cool dwarf stars. However, considering that all stars born more than $2 \mathrm{Gyr}$ ago with masses larger than $\sim 1.5 \mathrm{M}_{\odot}$ are now white dwarfs cooler than $10000 \mathrm{~K}$, our colour selection was excluding a significant population of these objects. We still limited our classification to spectra with $\mathrm{S} / \mathrm{N}_{g} \geq 3-7$, depending on the spectral type - down to lower $\mathrm{S} / \mathrm{N}_{g}$ for DA stars because hydrogen lines are stronger and easier to detect, but to higher $\mathrm{S} / \mathrm{N}_{g}$ in other classes.

\subsection{Spectral classification}

DR14 uses improved flux calibration, with atmospheric differential refraction corrected on a per exposure basis following the recipe described in Margala et al. (2016), and improved co-addition of individual exposures. The Stellar Parameters Pipeline, which we used for our initial spectral class selection, are from Lee et al. (2008a,b) and Allende Prieto et al. (2008).

The wavelength coverage is from 3800 to $9200 \AA$ for the SDSS spectrograph (up to Plate 3586 ), and 3650 to $10400 \AA$, for the BOSS spectrograph, with a resolution of 1500 at $3800 \AA$ and 2500 at $9000 \AA$, and a wavelength calibration better than $5 \mathrm{~km} \mathrm{~s}^{-1}$. All the spectra used in our analysis were processed with the spectroscopic reduction pipeline version v5_10_0 for BOSSS/SEQUELS/eBOSS, the spectroscopic reduction pipeline version 26 for the SDSS Legacy and SEGUE-1 programs, the special SDSS pipeline version 103 to handle stellar cluster plates, and the pipeline version 104 run on SEGUE-2 plates. These RUN2D numbers denote the version of extraction and redshift-finding code used. In all SDSS spectral line descriptions, vacuum wavelengths are used. The wavelengths are shifted such that measured velocities are relative to the Solar system barycentre at the mid-point of each 15-min exposure.

Because we are interested in obtaining accurate mass distributions for our DA and DB stars, we were conservative in labelling a spectrum as a clean DA or DB, adding additional subtypes and uncertainty notations (:) if we saw signs of other elements, unresolved companions, or magnetic fields $(\mathrm{H})$ in the spectra. While some of our mixed white dwarf subtypes would probably be identified as clean DAs or DBs with better S/N spectra, few of our identified clean DAs or DBs would likely be found to have additional spectral features within our detection limit.

We looked for the following features to aid in the classification for each specified white dwarf subtype:

(i) Balmer lines - normally broad and with a steep Balmer decrement (DA but also DAB, DBA, DZA, and subdwarfs)

(ii) He I $4471 \AA$ (DB, subdwarfs)

(iii) He II $4686 \AA$ (DO, PG1159, sdO)

(iv) $\mathrm{C} 2 \mathrm{Swan}$ band or atomic $\mathrm{C}$ I lines (DQ)

(v) Ca II H \& K (DZ, DAZ, DBZ)

(vi) C II $4367 \AA$ (HotDQ)

(vii) Zeeman splitting (magnetic white dwarfs)

(viii) featureless spectrum with significant proper motion (DC)

(ix) flux increasing in the red (binary, most probably M companion)

(x) O I 6158, 7774, $8448 \AA$ (DS, oxygen dominated)

(xi) $\mathrm{H}$ and $\mathrm{He}$ emission lines (CVs and $\mathrm{M}$ dwarf companions) 
Table 1. Classification of 37053 spectra in Table 2.

\begin{tabular}{ll}
\hline Number & Type \\
\hline 15716 & DA \\
1358 & DB \\
1847 & DC \\
524 & DQ \\
598 & DZ \\
45 & DO/PG $1159 / \mathrm{O}(\mathrm{He}) / \mathrm{O}(\mathrm{H})$ \\
210 & sdB \\
205 & sdO \\
311 & CV \\
4 & DS \\
1 & DH \\
14 & BHB \\
15855 & sdA \\
447 & dC \\
8 & BL LAC \\
\hline
\end{tabular}

Table 1 is a tally of the 37053 objects we classified in Table 2. As 15716 objects were classified by us as DAs and 1358 as DBs, of the 20088 white dwarfs in the table, 78 per cent are DAs.

Among the 15716 DAs, we found 474 magnetic DAHs, 598 unresolved binaries with main-sequence $\mathrm{M}$ dwarf companions $(\mathrm{DA}+\mathrm{M}), 136 \mathrm{DAZs}$ with $\mathrm{Ca}$ and/or $\mathrm{Mg}$ lines, and $52 \mathrm{DABs}$ contaminated by $\mathrm{He}$ I lines. We also found 41 stars having an extremely steep Balmer decrement (i.e. only a broad $\mathrm{H} \alpha$ and sometimes $\mathrm{H} \beta$ is observed while the other lines are absent) that could not be fit with a pure hydrogen grid (see Section 2.3 below), or indicated extremely high gravities. We find that these objects are best explained as helium-rich DAs, and therefore with an extremely thin $\mathrm{H}$ layer mixed with the underlying $\mathrm{He}$, and denote them $\mathrm{DA}(\mathrm{He})$.

We classified 447 spectra as dC - dwarf carbon stars, in line with Green (2013) and Farihi et al. (2018). We cannot identify a clear visual discontinuity from the coolest DQs to the hottest $\mathrm{dCs}$ either in term of the $\mathrm{C}$ line strength or the colour-magnitude diagram (see Fig. 13). We do not have spectral models for dCs, so we do not determine their properties. Of the $340 \mathrm{CVs}, 9$ are AM CVn type, with pure $\mathrm{He}$ spectra, and $87 \mathrm{CV}$ s show both $\mathrm{H}$ and $\mathrm{He}$ lines. As an example of the spectra of $\mathrm{CVs}$ found in our search, Fig. 1 shows the spectrum of the ultracompact white dwarf binary AM CVn SDSS J141118.31+481257.66, with $g=19.38$, spectrum P-M-F 1671-53446-0010, with He emission double lines (Fig. 1). Rivera Sandoval \& Maccarone (2018) reported an outburst, the first recorded for this star. Ramsay et al. (2018) review of AM CVns show many have outbursts reported; AM CVn are ultracompact hydrogen-deficient binaries, each consisting of a white dwarf accreting helium-dominated material from a degenerate or semidegenerate donor star.

We classified 15793 stars as sdAs, stars with spectra dominated by narrow hydrogen lines, following Kepler et al. (2016). Solar metallicity main-sequence A stars have absolute magnitudes $M_{g} \simeq$ $0-2$. As stars brighter than $g=14.5$ saturate in SDSS, only A stars with distance moduli larger than 12.5 are observed in SDSS, i.e. farther than $3.5 \mathrm{kpc}$. Because SDSS observed mainly perpendicular to the disc (galactic latitude in general larger than $30 \mathrm{deg}$ ), these would be located in the halo, where A stars should already have evolved off the main sequence. Thus, these sdA stars are mostly likely very low metallicity main-sequence stars $([\mathrm{Fe} / \mathrm{H}] \lesssim-1.0)$, whose spectra are dominated by hydrogen because they lack significant metals, and most have masses smaller than the Sun, or their spectra would show higher effective temperatures than observed. As their absolute magnitude, according to Gaia parallaxes, cover $-8 \geq M_{G} \geq 10$ (see Fig. 11), they cannot be classified as normal main-sequence A stars, presenting much lower masses and temperature than AV stars. They are hotter than sdF stars (Scholz et al. 2015). Some of these sdAs may be stars that lost mass due to binary interaction, resulting most probably in He core stars, precursors of ELMs, and ELMs (Pelisoli, Kepler \& Koester 2018a; Pelisoli et al. 2018b, 2019) (see Section 3.3).

\subsection{Models}

After classifying white dwarf and subdwarf stars, we fitted the observed spectra to improved models of pure DAs and DBs (Koester et al. 2011; Koester \& Kepler 2015), DOs (Reindl et al. 2014, 2014a; Reindl \& Rauch 2015), sdBs, and sdOs (Geier et al. 2015, 2017a,b). For DAs, we used ML2/ $\alpha=0.7$ models, with an LTE grid extending from $5000 \mathrm{~K} \leq T_{\text {eff }} \leq 80000 \mathrm{~K}$ and $3.5 \leq \log g \leq 9.5$ dex (cgs). For $T_{\text {eff }} \leq 14000 \mathrm{~K}$ we corrected the temperature and gravity to the 3D calculations of Tremblay et al. (2013), resulting in a flat $\log g$ distribution down to $T_{\text {eff }} \simeq 10000 \mathrm{~K}$, as shown in Fig. 2. The figure also show models for He core pre-white dwarfs (Althaus et al. 2015; Istrate et al. 2016) and for the ZAHB to show the region where we do not consider the objects as white dwarfs. For DAs whose LTE analysis indicates $T_{\text {eff }} \geq 45000 \mathrm{~K}$ (that is where NLTE effects become important), we employed NLTE models. We computed a pure $\mathrm{H}$ grid with the Tübingen non-LTE Model-Atmosphere Package (TMAP; Rauch \& Deetjen 2003; Werner et al. 2003, 2012) spanning from $T_{\text {eff }}=40000-200000 \mathrm{~K}$ (step size $5000 \mathrm{~K}$ for $T_{\text {eff }}<$ $100000 \mathrm{~K}$ and $10000 \mathrm{~K}$ for $T_{\text {eff }}>100000 \mathrm{~K}$ ) and $\log g=6.0-9.0$ (step size $0.5 \mathrm{dex}$ ). To calculate synthetic line profiles, we used Stark line-broadening tables provided by Tremblay \& Bergeron (2009). To derive the effective temperatures and surface gravities the Balmer lines of the hot DAs were fitted in an automated procedure by means of $\chi^{2}$ minimization using the FITSB2 routine (Napiwotzki 1999) and calculated the statistical $1 \sigma$ errors. Each fit was then inspected visually to ensure the quality of the analysis. We excluded hot DAs whose spectra show an red excess and/or central emission features in the Balmer lines that cannot be the result of NLTE effects but are likely due the to the irradiation of a cool companion by the hot white dwarf.

Fig. 3 shows the histogram of the number of DA stars versus effective temperature. The hottest DAs we analysed have $T_{\text {eff }} \simeq$ $120000 \mathrm{~K}$. The decrease of the number in the coolest bin is mainly due to incompleteness, because cooler stars are fainter partially compensated by the low-mass stars that are brighter, but also affected by the finite age of the disc stars (Winget et al. 1987).

For DBs we use ML2/ $\alpha=1.25$ LTE models as in Koester \& Kepler (2015), with $12000 \mathrm{~K} \leq T_{\text {eff }} \leq 45000 \mathrm{~K}$, and $7 \leq \log g \leq$ $9.5 \mathrm{dex}$ (cgs), resulting in the $T_{\text {eff }}-\log g$ distribution shown in black in Fig. 4. An increase in the estimated log $g$ can be seen for $T_{\text {eff }} \lesssim 16000 \mathrm{~K}$. This is not solved when pure He 3D corrections are applied (Cukanovaite et al. 2018), shown in red in Fig. 4, and is probably caused by poor estimates of neutral broadening.

Because the spectral fits are normally degenerate between a hot solution(s) and a cool one, we also fitted the ugriz colours of DAs and DBs to synthetic colours derived from the same atmospheric models, and used the photometric values to guide our spectral parameter determinations. For DCs, DQs, and DZs, we only estimated their $T_{\text {eff }}$ from the colours derived from the atmospheric models of Koester (2010). 


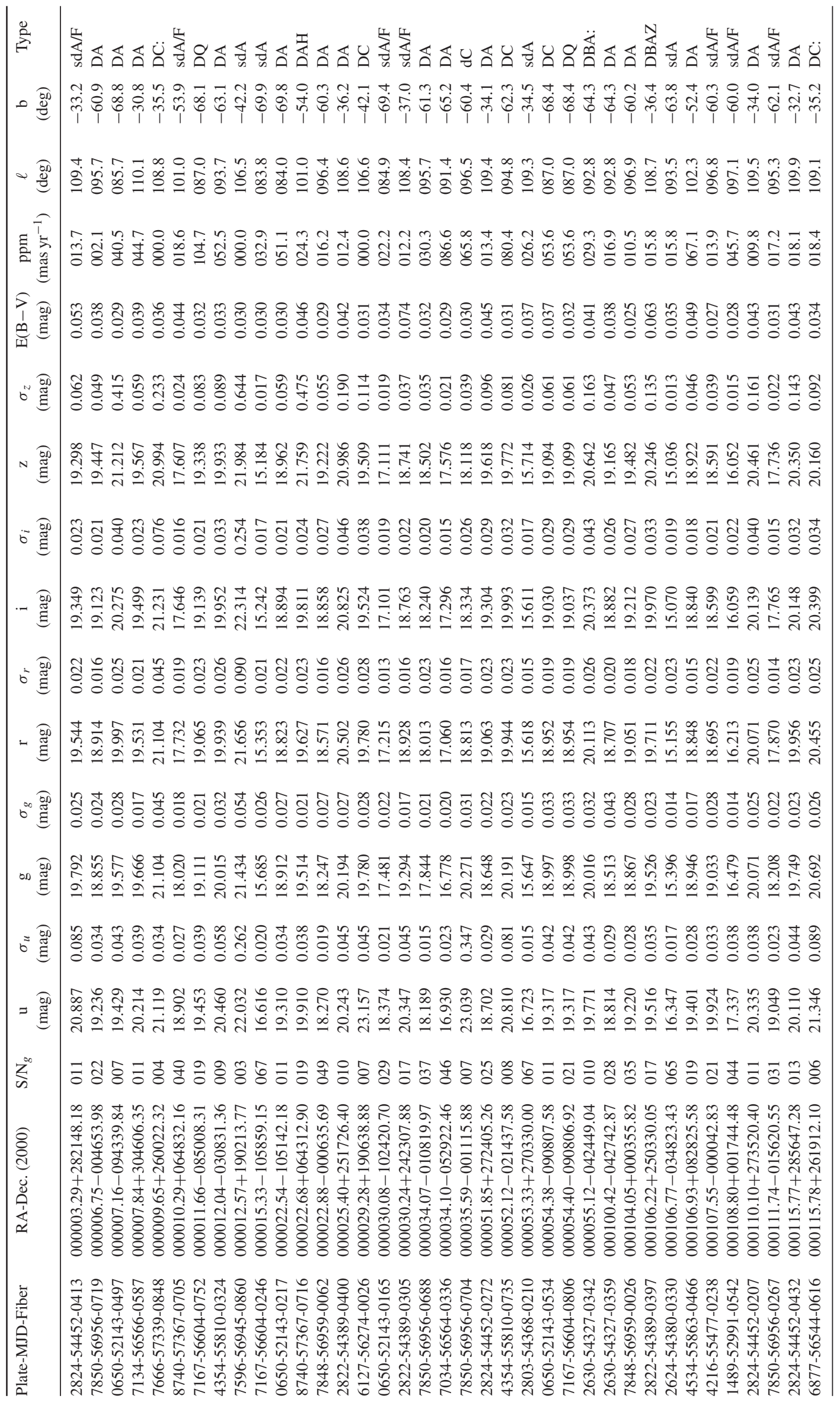




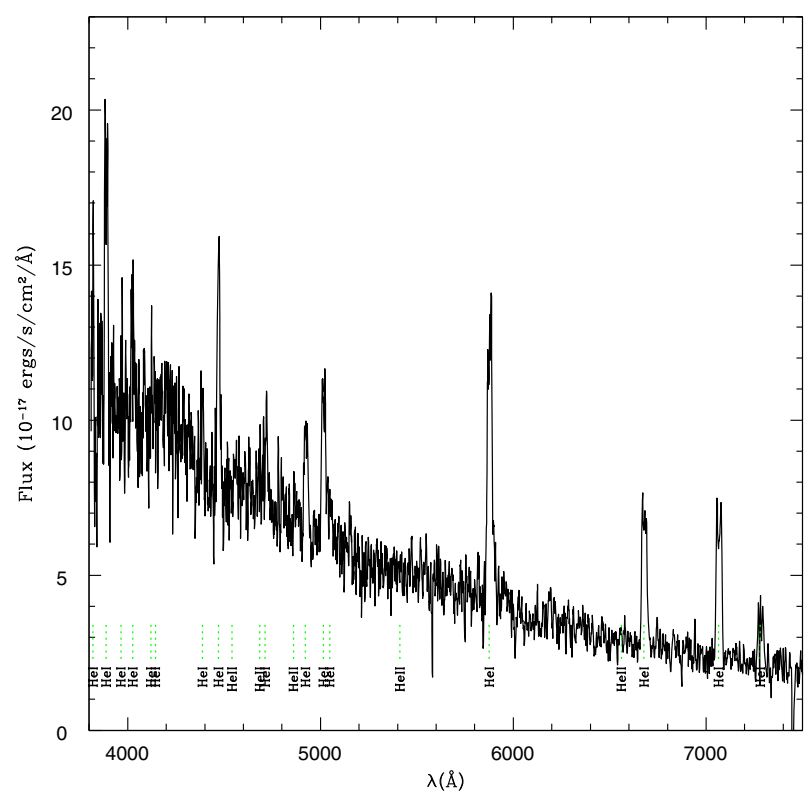

Figure 1. Spectrum of the outbursting AM CVn SDSS J141118.31+481257.66, with He emission double lines.

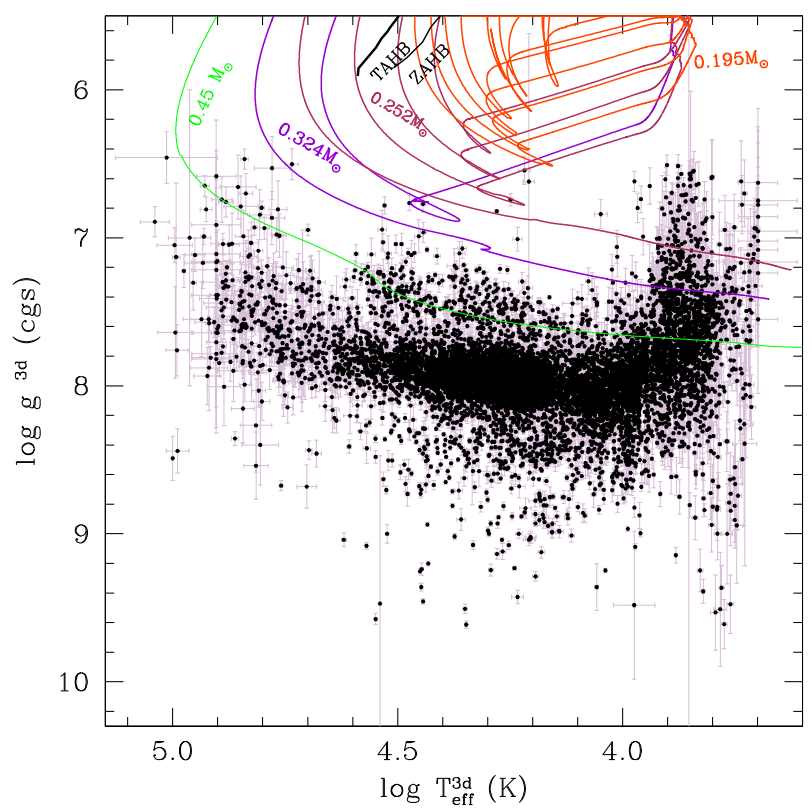

Figure 2. Surface gravity $(\log g)$ and effective temperature ( $\left.T_{\text {eff }}\right)$ estimated for the 10189 DA white dwarf stars for which the SDSS spectra has $\mathrm{S} / \mathrm{N}_{g}>10$, after applying three-dimensional convection atmospheric model corrections from Tremblay et al. (2013), in black. The ZAHB plotted was calculated with solar composition models. These delimit the region of solar metallicity Blue Horizontal Branch stars. It indicates the highest possible surface gravity for a hot subdwarf. Stars with $T_{\text {eff }} \leq 45000 \mathrm{~K}$ and smaller surface gravities than the ZAHB are sdBs. We have also plotted 0.45, 0.3, 0.2, and $0.15 \mathrm{M}_{\odot}$ models of He core pre-white dwarfs (Althaus et al. 2015; Istrate et al. 2016) to guide the eye to the limiting region of what we call white dwarfs.

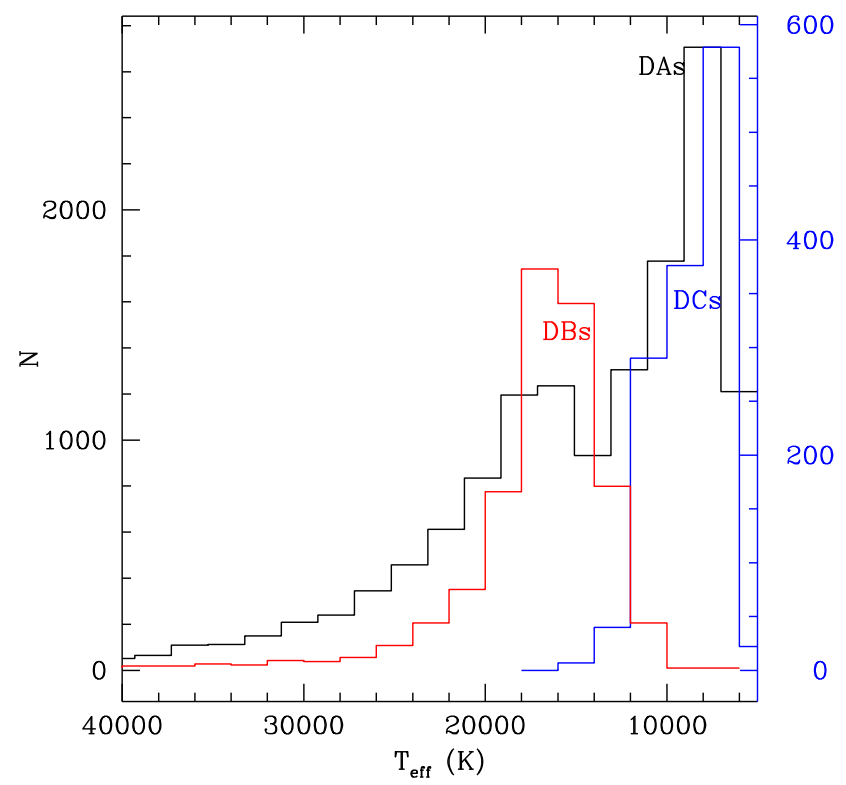

Figure 3. Histogram of the number of DA stars versus effective temperature (in black), compared to the distribution for DCs (in blue), and DBs (in red). The number scale for DCs and DBs is shown on the right.

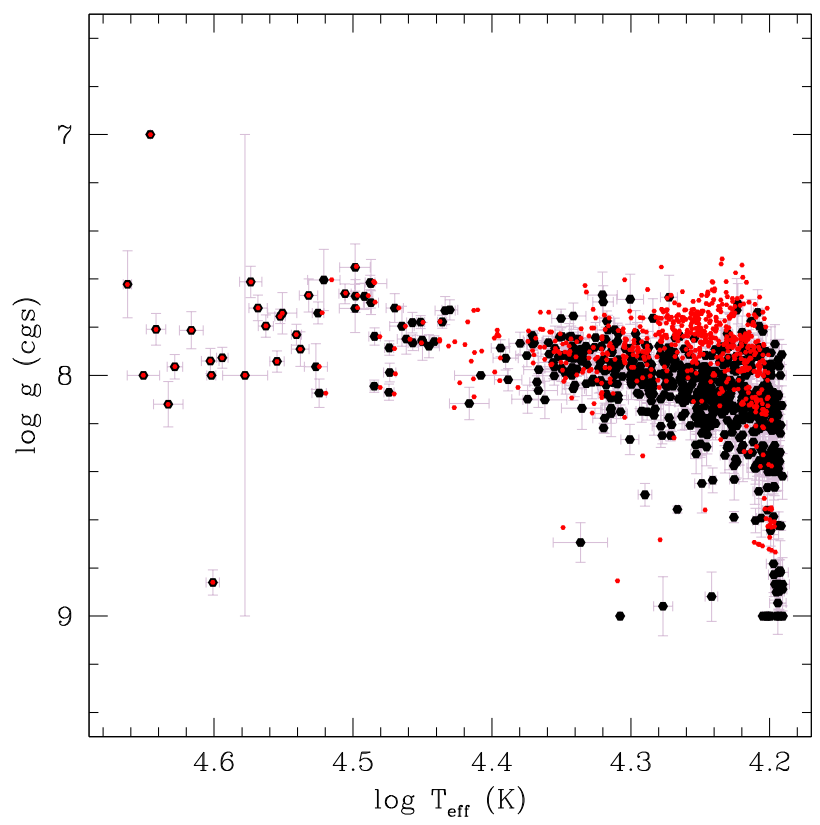

Figure 4. Surface gravity $(\log g)$ and effective temperature $\left(T_{\text {eff }}\right)$ estimated for $805 \mathrm{DB}$ white dwarf stars with spectral $\mathrm{S} / \mathrm{N}_{g} \geq 10$. The increase in apparent gravity below $T_{\text {eff }} \simeq 16000 \mathrm{~K}$ is probably caused by incorrect neutral broadening estimative (Schaeuble et al. 2017). In red are the values after applying the pure He 3D corrections of Cukanovaite et al. (2018).

\section{RESULTS}

\subsection{Masses}

For white dwarfs, the main indicator of $\log g$ is the width of the atmospheric absorption lines. However, for $T_{\text {eff }}<10000 \mathrm{~K}$, the width of the hydrogen lines becomes very weakly dependent on gravity. As a result, it is very difficult to distinguish low-mass 


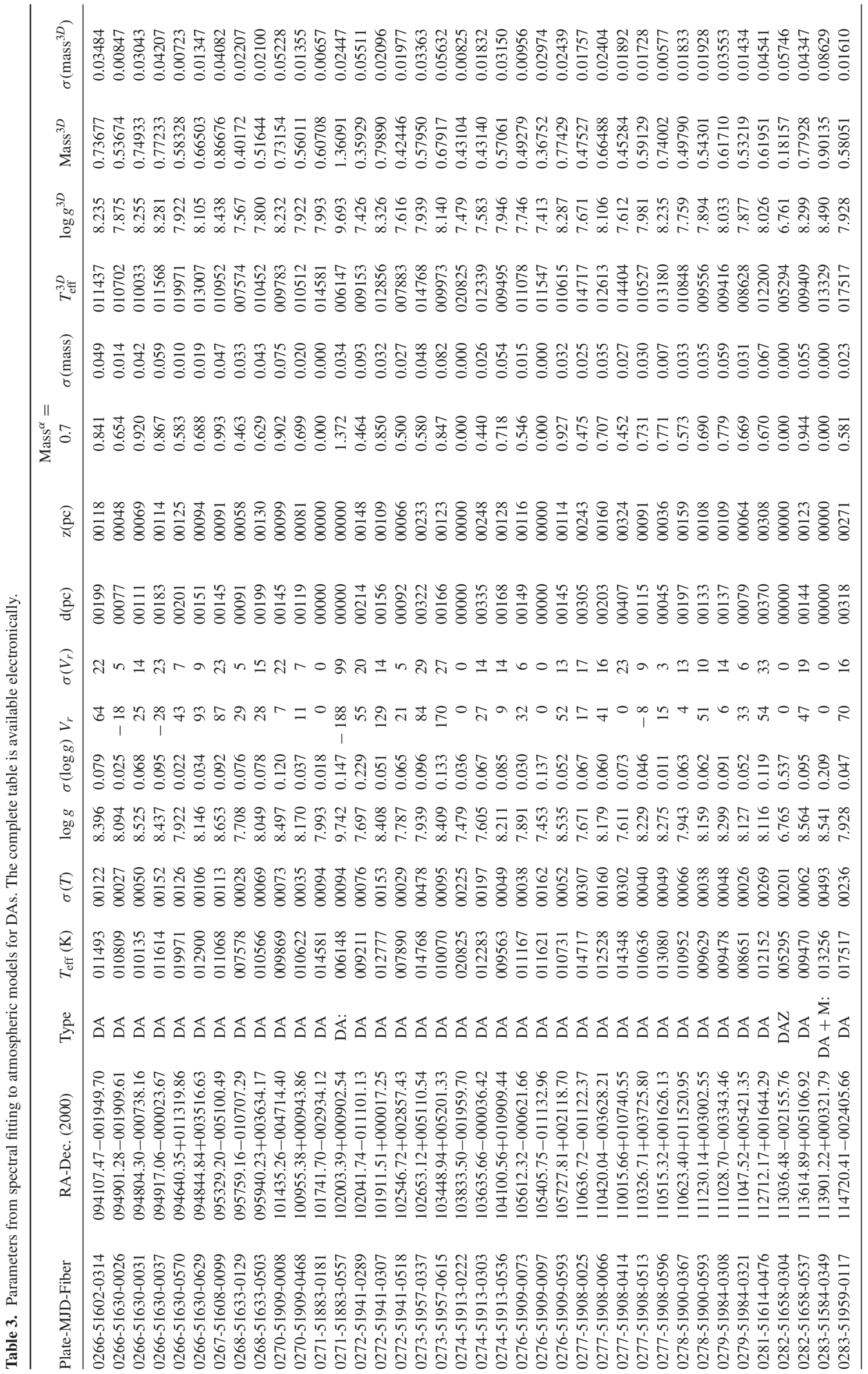




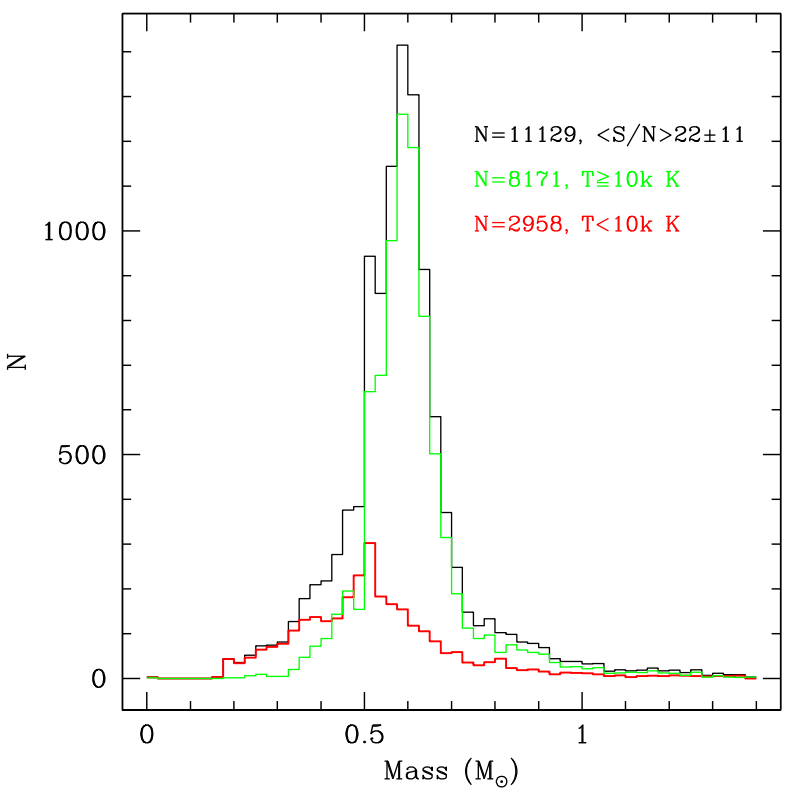

Figure 5. Mass distribution for the $11129 \mathrm{DAs}$ with $\mathrm{S} / \mathrm{N}_{g} \geq 10$. This sample overall shows a mean mass of $\langle M\rangle=0.5904 \pm 0.0014 \mathrm{M}_{\odot}$. For stars with $T_{\text {eff }}>10000 \mathrm{~K}$, however, the mean mass is $\langle M\rangle=0.6131 \pm 0.0014 \mathrm{M}_{\odot}$, whereas for stars with $T_{\text {eff }}<10000 \mathrm{~K}$, the mean mass is $\left\langle M_{\mathrm{DB}}\right\rangle=0.5276 \pm$ $0.0035 \mathrm{M}_{\odot}$. Most of the low-mass DAs concentrate below $T_{\text {eff }}=10000 \mathrm{~K}$.

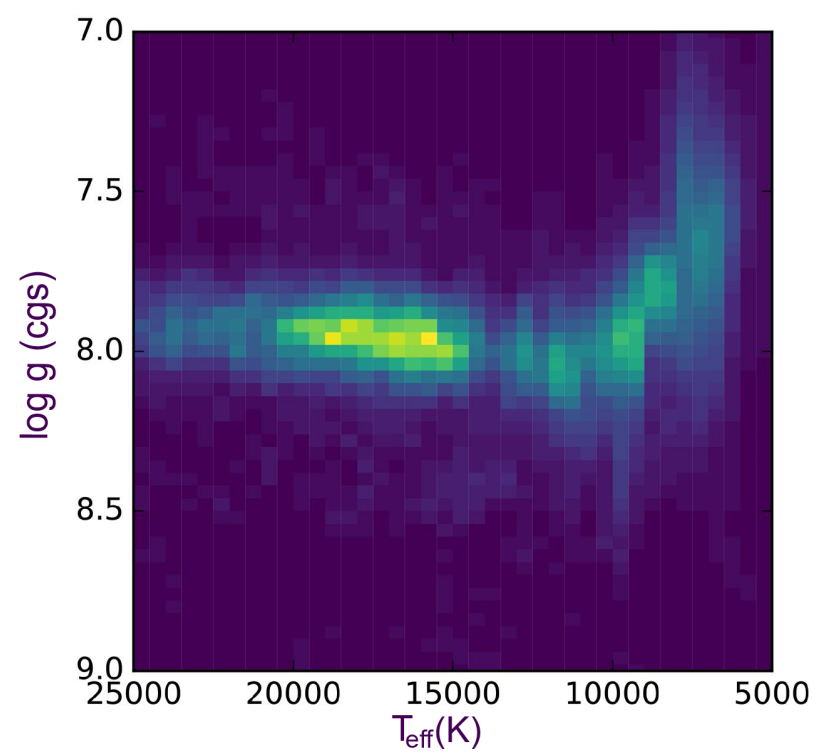

Figure 6. Hess diagram - density distribution - across effective temperature and surface gravity for DAs with spectra with $\mathrm{S} / \mathrm{N} \geq 10$, showing the lowmass DA white dwarfs concentrate below $T_{\text {eff }}=10000 \mathrm{~K}$.

Table 4. Distribution of DAs with $T_{\text {eff }}$.

\begin{tabular}{lc}
\hline Number & Temperature range \\
\hline 273 & $<6000 \mathrm{~K}$ \\
2938 & $6000-8000 \mathrm{~K}$ \\
2312 & $8000-10000 \mathrm{~K}$ \\
3325 & $10000-15000 \mathrm{~K}$ \\
3179 & $15000-20000 \mathrm{~K}$ \\
2968 & $20000-40000 \mathrm{~K}$ \\
596 & $>40000 \mathrm{~K}$ \\
15591 & Total \\
\hline
\end{tabular}

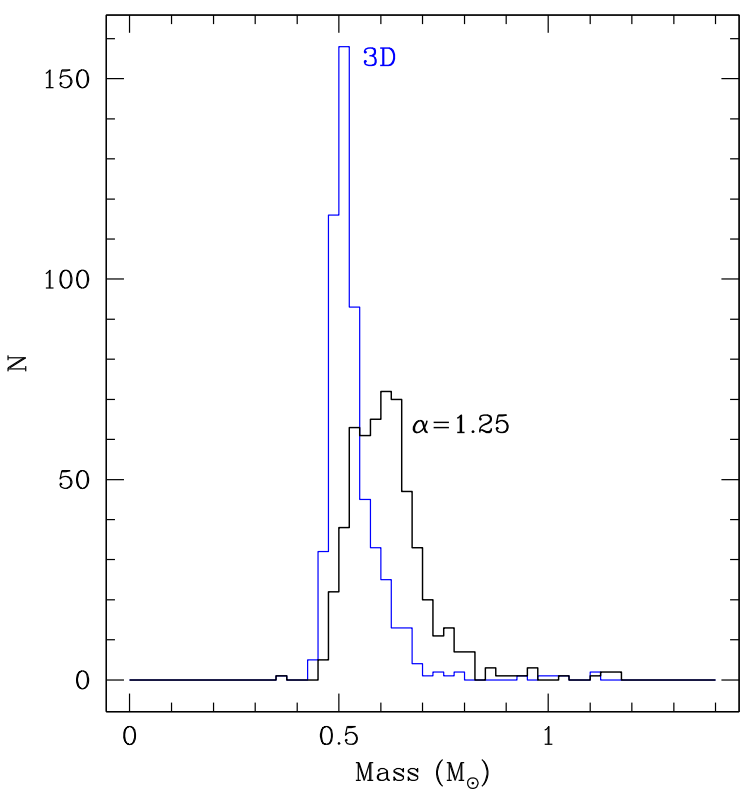

Figure 7. Mass distribution for the 550 pure DBs with $\mathrm{S} / \mathrm{N}_{g} \geq 10$ with $T_{\text {eff }}>16000 \mathrm{~K}$, the distribution shows a mean mass of $\left\langle M_{\mathrm{DB}}^{\alpha=1.25}\right\rangle=$ $0.618 \pm 0.004 \mathrm{M}_{\odot}$, and a dispersion of $0.098 \mathrm{M}_{\odot}$. With the pure He 3D correction, in blue, the mean mass decreases to $\left\langle M_{\mathrm{DB}}^{3 \mathrm{D}}\right\rangle=0.536 \pm 0.003 \mathrm{M}_{\odot}$, with a dispersion of $0.074 \mathrm{M}_{\odot}$. For lower temperatures the $\log g$, and therefore mass, is not trustworthy due to large uncertainties in the neutral broadening estimative. The DB mass distribution does not extend to masses below $0.45 \mathrm{M}_{\odot}$ or masses above $1.1 \mathrm{M}_{\odot}$; however, the statistics is much poorer than for DAs. The average S/N of the spectra is $\left\langle S / N_{g}\right\rangle=25$.

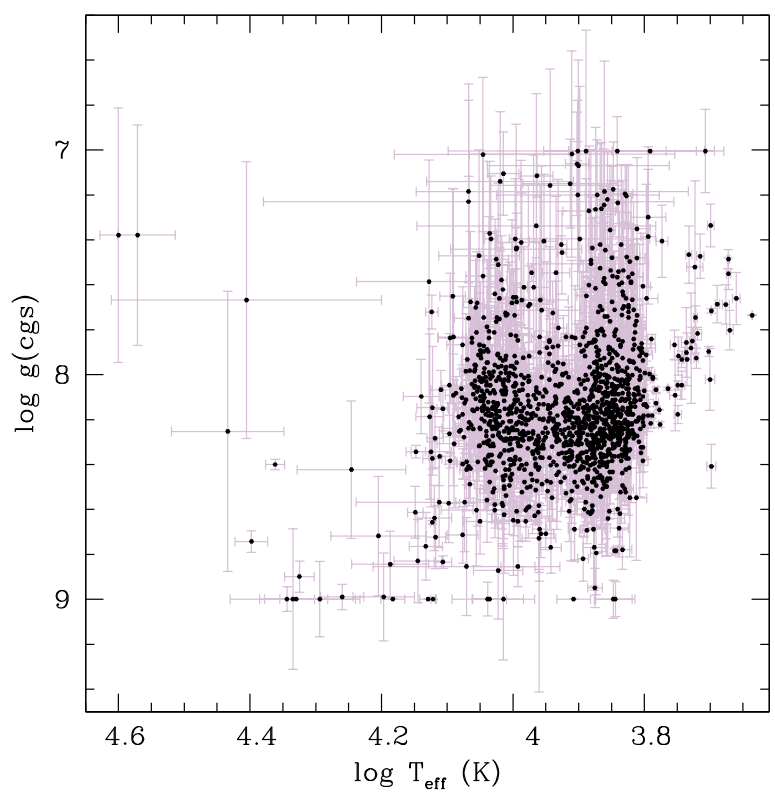

Figure 8. Surface gravity $(\log g)$ for the DCs, obtained from the SDSS ugriz photometry, Gaia DR2 parallax, and a He-atmosphere mass-radius relation.

white dwarfs and metal-poor main-sequence $\mathrm{A} / \mathrm{F}$ stars in the $T_{\text {eff }}<$ $10000 \mathrm{~K}$ and $\log g<6.5$ range solely with visual inspection, even though low-metallicity main-sequence stars have an upper limit to $\log g \lesssim 4.64$, for a turn-off mass of $\sim 0.85 \mathrm{M}_{\odot}$. The two steps we took to overcome this limitation was the extension of the model 


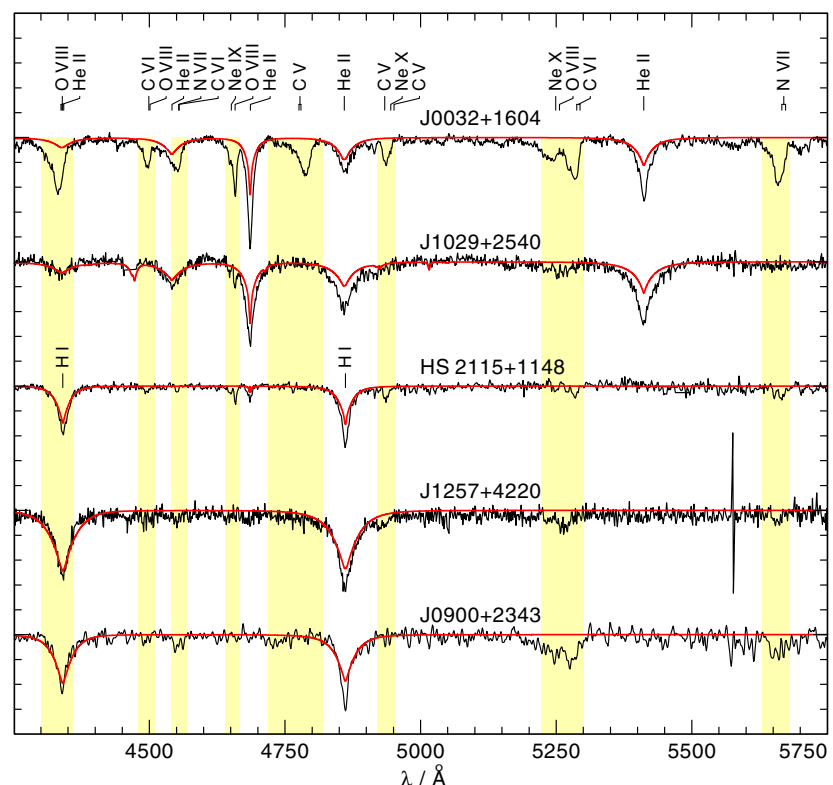

Figure 9. Normalized SDSS spectra of the newly discovered DO (upper two) and DA (bottom two) hot wind white dwarfs. The spectrum of SDSS J090023.89+234353.2 is convolved with a Gaussian (FWMH $=3$ $\AA$ ) to smooth out the noise. The TWIN spectrum of HS $2115+1148$, for more than $20 \mathrm{yr}$ the only known H-rich hot wind white dwarf, is shown for comparison.

grid to $\log g \geq 3.5$, fitting all the spectra we classified as DAs and sdAs, using the result to separate $\log g \geq 6.5$ as white dwarfs, and finally, after Gaia DR2, using the parallaxes, as discussed in Section 4.

Kleinman et al. (2013) limited the white dwarf classification to surface gravity $\log g \geq 6.5$. At the cool end of our sample, $\log g=$ 6.5 corresponds to a mass around $0.2 \mathrm{M}_{\odot}$, well below the single mass evolution in the lifetime of the Universe - but reachable via interacting binary evolution. The He-core white dwarf stars in the mass range $0.2-0.45 \mathrm{M}_{\odot}$, referred to as low-mass white dwarfs, are usually found in close binaries, often double degenerate systems (Marsh, Dhillon \& Duck 1995), being most likely a product of interacting binary stars evolution. More than 70 percent of those studied by Kilic et al. (2011) with masses below $0.45 \mathrm{M}_{\odot}$ and all but a few with masses below $0.3 \mathrm{M}_{\odot}$ show radial velocity variations (Brown et al. 2013; Gianninas et al. 2014; Brown, Kilic \& Gianninas 2017). Kilic et al. (2007) suggest single low-mass white dwarfs result from the evolution of old metal-rich stars that truncate evolution before the helium flash due to severe mass-loss. They also conclude all white dwarfs with masses below $\simeq 0.3 \mathrm{M}_{\odot}$ must be a product of binary star evolution involving interaction between the components.

The spectroscopic sdA sample defined in Kepler et al. (2016) and used in our pre-selection here, being only a visual determination of narrow $\mathrm{H}$ lines and absence of strong metal lines, includes many types of objects: real white dwarfs, ELMs, preELMs, low-metallicity main-sequence stars, and even giants with low atmospheric metallicity. We need to define separate classes depending on the absolute luminosity (radius) to distinguish among them (see Section 3.3). Even though the nomenclature would suggest that subdwarfs have smaller radii than main-sequence stars, it is not always the case - they mainly have smaller masses.

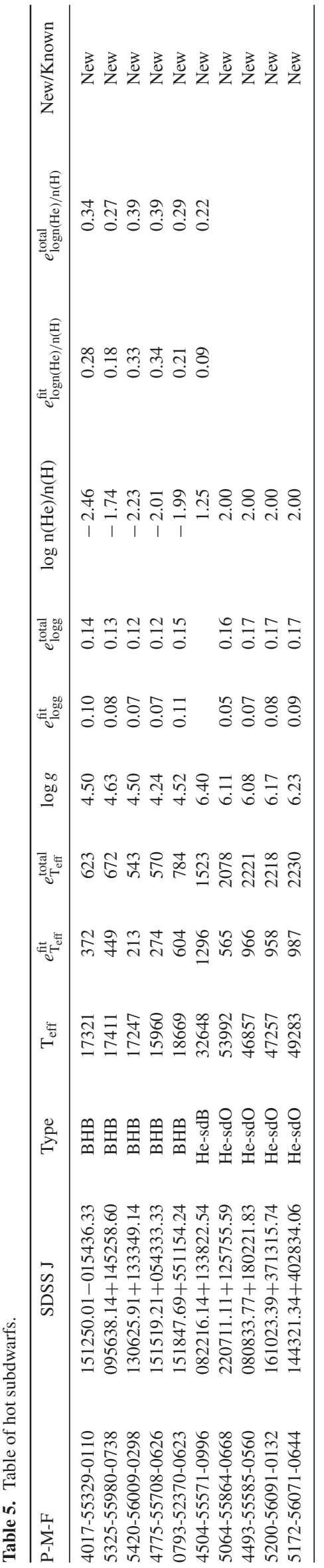




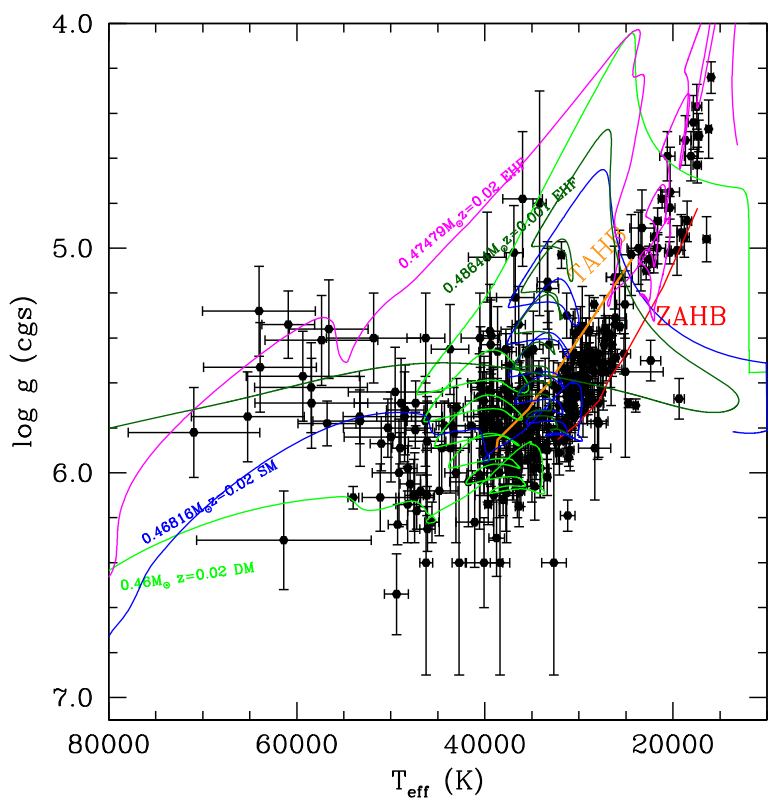

Figure 10. Hot subdwarfs, sdOs and sdBs. The ZAHB and the Terminal Age Horizontal Branch (TAHB) plotted were calculated with solar composition models. In green we also plot a $0.46816 \mathrm{M}_{\odot}, z=0.02$, SM (shallow mixing hot flasher), DM (deep mixing hot flasher), and EHF (early hot flasher) models from Battich et al. (2018).

Table 3 shows the atmospheric parameters obtained from the fitting of the spectra for DAs. Fig. 5 shows the mass distribution for DAs with $\mathrm{S} / \mathrm{N}_{g} \geq 10$, with 11129 stars, and result in a mean mass $\left\langle M_{\mathrm{DA}}\right\rangle=0.5903 \pm 0.0014 \mathrm{M}_{\odot}$, and individual dispersion of $0.152 \mathrm{M}_{\odot}$. For the $8171 \mathrm{DAs}$ with $T_{\text {eff }} \geq 10000 \mathrm{~K}$, the mean mass is $\left\langle M_{\mathrm{DA}}\right\rangle=0.6131 \pm 0.0014 \mathrm{M}_{\odot}$, with a dispersion $0.126 \mathrm{M}_{\odot}$, while for those 2958 with $T_{\text {eff }}<10000 \mathrm{~K},\left\langle M_{\mathrm{DA}}\right\rangle=0.5276 \pm$ $0.0035 \mathrm{M}_{\odot}$ with a dispersion $0.174 \mathrm{M}_{\odot}$. Fig. 6 shows the density of DAs versus temperature and surface gravity, showing the surface gravity decreases significantly below $T_{\text {eff }} \simeq 10000 \mathrm{~K}$.

Table 4 shows the effective temperature distribution for DAs in our sample that were found to have effective temperatures in the range specified. The hottest we found is the DAO SDSS J160828.69+422101.77, with an $\mathrm{S} / \mathrm{N}_{g}=43$ spectrum and $T_{\text {eff }}=120000 \pm 10000 \mathrm{~K}$, while the hottest pure DA is SDSS J101756.24+411524.72, with an $\mathrm{S} / \mathrm{N}_{g}=26$ spectrum and $T_{\text {eff }}=110000 \pm 8000 \mathrm{~K}$. The most massive pure DAs are SDSS J121234.85+165320.26, with an $\mathrm{S} / \mathrm{N}=17$ spectrum and $T_{\text {eff }}=5944 \pm 91 \mathrm{~K}, \log g=9.611 \pm 0.166, M=1.370 \pm$ $0.006 \mathrm{M}_{\odot}$, and SDSS J152958.12+130454.80, with an $\mathrm{S} / \mathrm{N}_{g}=$ 48 spectrum and $T_{\text {eff }}=5758 \pm 99 \mathrm{~K}, \log g=9.476 \pm 0.197$, $M=1.364 \pm 0.005 \mathrm{M}_{\odot}$. We caution that the quoted uncertainties are only the internal uncertainties from the least-square fits. For stars with multiple spectra, our mean external uncertainty is 5 percent in the effective temperature and $0.05 \mathrm{dex}$ in $\log g$, but for $T_{\text {eff }} \leqslant$ $10000 \mathrm{~K}$, the real uncertainty is unknown.

The histogram of the number of DB stars versus effective temperature can be seen in Fig. 3 (in red), compared to for DAs (in black). We see no obvious DB gap, just the normal decrease in DBs hotter than $30000 \mathrm{~K}$ due to the ionization of He I.

Fig. 7 shows the mass distribution for the 550 pure DBs with $\mathrm{S} / \mathrm{N}_{g} \geq 10$ spectra and $T_{\text {eff }} \geq 16000 \mathrm{~K}$, with and without the pure $\mathrm{He}$ 3D convection correction following Cukanovaite et al. (2018). Without the correction, the mean mass is $\left\langle M_{\mathrm{DB}}^{\alpha=1.25}\right\rangle=$
$0.618 \pm 0.004 \mathrm{M}_{\odot}$, and a dispersion of $0.098 \mathrm{M}_{\odot}$. With the $3 \mathrm{D}$ correction, the mean mass decreases to $\left\langle M_{\mathrm{DB}}^{3 \mathrm{D}}\right\rangle=0.536 \pm 0.003 \mathrm{M}_{\odot}$, and a dispersion of $0.074 \mathrm{M}_{\odot}$. The theoretical neutral broadening used in the models overestimates the $\log g$, and therefore masses, for lower temperatures (e.g. Koester \& Kepler 2015; Schaeuble et al. 2017). For the 333 pure DB with $S / \mathrm{N}_{g} \geq 20$, we obtain $\left\langle M_{\mathrm{DB}}^{3 \mathrm{D}}\right\rangle=0.533 \pm 0.003 \mathrm{M}_{\odot}$, with a dispersion of $0.058 \mathrm{M}_{\odot}$, i.e. the $\mathrm{S} / \mathrm{N}$ is not changing the mean value. The low mean mass is a direct consequence of the pure $\mathrm{He} 3 \mathrm{D}$ corrections. Our fitted mean surface gravity, with the ML2/ $\alpha=1.25$ models is $\log g=$ $8.032 \pm 0.008$, while the 3D corrected $\log g=7.864 \pm 0.007$. A similar mean mass for DBs was obtained by Genest-Beaulieu \& Bergeron (2019).

The two highest mass DBs, above $16000 \mathrm{~K}$, are SDSS J163757.58+190526.01, with $\mathrm{S} / \mathrm{N}_{g}=24, \quad T_{\text {eff }}=$ $39895 \pm 441 \mathrm{~K}, \log g=8.86 \pm 0.05, M=1.111 \pm 0.017 \mathrm{M}_{\odot}$, and SDSS J081223.85+254842.82, with $\mathrm{S} / \mathrm{N}_{g}=14, T_{\text {eff }}=20394 \pm$ $1000 \mathrm{~K}, \log g=8.85 \pm 0.05, M=1.100 \pm 0.005 \mathrm{M}_{\odot}$, but our models, prior to the $3 \mathrm{D}$ correction, only go up to $\log \mathrm{g}=9.0$.

For the 1314 DCs in Table 2 with Gaia DR2 parallaxes, we obtain their masses from the ugriz, colours and Gaia DR2 parallax, following Ourique et al. (2019). Their radius is estimated from the observed flux and distance, assuming an He atmosphere massradius relation. Their distribution shows a mean surface gravity $\left\langle\log g^{\mathrm{DC}}\right\rangle=8.166 \pm 0.007 \mathrm{dex}$ (cgs), with a dispersion of $0.245 \mathrm{dex}$, and a mean mass of $\left\langle M^{\mathrm{DC}}\right\rangle=0.694 \pm 0.004 \mathrm{M}_{\odot}$, with a dispersion of $0.127 \mathrm{M}_{\odot}$.

Fig. 8 shows the effective temperature and surface gravity for 1314 DCs with Gaia DR2 parallaxes and ugriz SDSS colours. The mean mass obtained is $\langle M\rangle_{\mathrm{DC}}=0.694 \pm 0.004 \mathrm{M}_{\odot}$ with a dispersion of $0.127 \mathrm{M}_{\odot}$. Ourique et al. (2019) presented the first DC mass distribution, using the Gaia colours and distances, showing it concentrates at higher masses than DBs. It is unlikely caused by an increase in the pressure by undetected hydrogen dredged up affecting the colours, as the non-DA to DA ratio increases below $16000 \mathrm{~K}$.

\subsection{Hot white dwarfs}

We identified spectroscopically in our sample a total of 12 PG 1159 and $\mathrm{O}(\mathrm{He})$ stars and 36 DOs with spectra dominated by He II lines. Furthermore, we found one $\mathrm{O}(\mathrm{H})$ star (Reindl et al. 2016) and 48 DAO stars, with spectra showing both $\mathrm{H}$ and $\mathrm{He}$ II lines as well as 310 hot DAs, showing only $\mathrm{H}$ lines. All these stars are hotter than $T_{\text {eff }}=45000 \mathrm{~K}$, where NLTE effects are important in the spectral analysis. We note that the majority of these objects were already known and that our catalogue is far from being complete with respect to the hottest white dwarfs found in previous SDSS data releases. This is a consequence of the $3 \sigma$ proper motion criterion, because hot white dwarfs are intrinsically more luminous and are detected over larger distances, and the more distant ones will have small proper motions.

One of the PG 1159 stars and 10 of the DO white dwarfs belong to the group of the so-called hot wind white dwarfs (Werner et al. 1995), i.e. they show abnormally broad and deep He II lines with seven of them showing additionally ultrahigh excitation (uhe) absorption lines (e.g. O VIII). For the latter objects we introduce the sub-classification uhe, i.e. PG 1159uhe and DOuhe. Our sample includes two newly identified hot wind DO white dwarfs, SDSS J003213.13+160434.7, which shows the strongest uhe features detected in any hot wind white dwarf so far, and SDSS J102907.31+254008.3, which shows only abnormally broad 


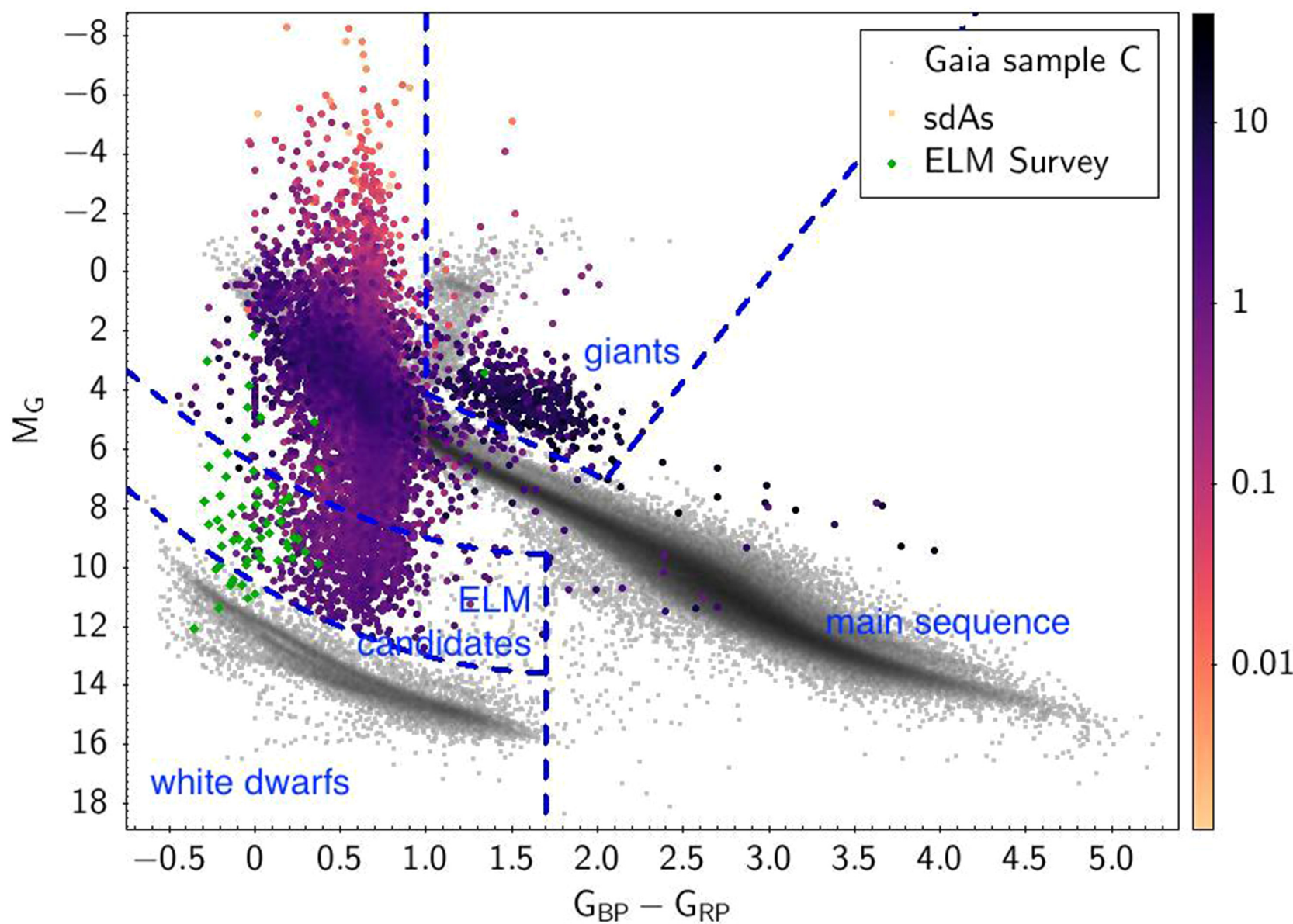

Figure 11. sdAs (colour-coded according to parallax_over_error) separated using their Gaia absolute magnitude and colour. They extend from the white dwarf cooling region, binary region, through the low-metallicity main sequence, to the giant phase. As a comparison, we also show the sample $\mathrm{C}$ of Lindegren et al. (2018), consisting of solar neighbourhood stars (within 100 pc) with clean parallax, and the ELMs of Brown et al. (2016) .

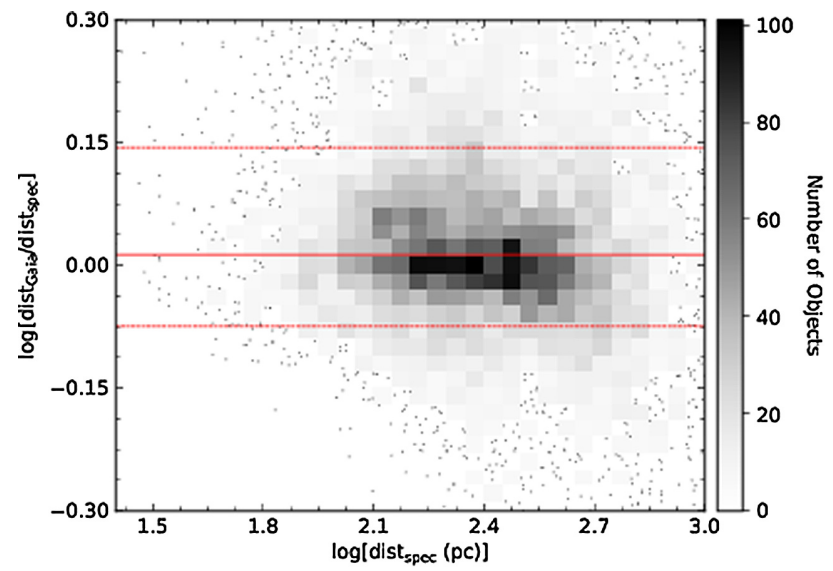

Figure 12. Distances for DA white dwarfs in our sample, estimated from the Gaia parallax uncertainty distribution, compared with the distance calculated from the spectroscopic distance modulus. The solid red line represents the median. The lower and upper dashed red lines represent, respectively, the 16 and 84 percentiles. The points represent bins with less than 5 objects. We did not use a Gaia DR2 parallax precision limit in this plot, or used the parallax to select the best spectroscopic solution. The distances from the parallax are compatible with the spectroscopic distances we obtained given the large error bars. and deep He II lines and possible an uhe feature located at $5250 \AA$. We also report the discovery of uhe features in two of the hot DA white dwarfs SDSS J090023.89+234353.2 and SDSS J125724.04+422054.2 (PG 1255+426). After more than 20 yr, these are the first two H-rich hot wind white dwarfs discovered since HS 2115+1148 (Dreizler et al. 1995). Fig. 9 shows the normalized SDSS spectra of the newly discovered objects. The uhe lines were recently shown to originate from an extremely hot, windfed circumstellar magnetosphere (Reindl et al. 2019). The two newly discovered DA hot wind white dwarfs show the Balmer line problem (failure to achieve a consistent fit to the Balmer lines, Werner 1996), which is also present in HS $2115+1148$. Thus, the Balmer line problem can serve as a first indicator for the hot wind phenomenon. It is assumed that the cooler parts of the magnetosphere constitute an additional line forming region of the too-broad and too-deep H I/-He II lines (Reindl et al. 2019).

\subsection{Subdwarfs}

We classified 77 stars as hot subdwarf sdOs, 128 sdOBs and 209 sdBs. To refine the visual classification and derive the atmospheric parameters, a quantitative spectral analysis was performed for all $\mathrm{sdO} / \mathrm{B}$ candidates in our sample with data of sufficient quality $\left(\mathrm{S} / \mathrm{N}_{g}\right.$ $>20$ ) and no atmospheric parameter determination in the literature. 


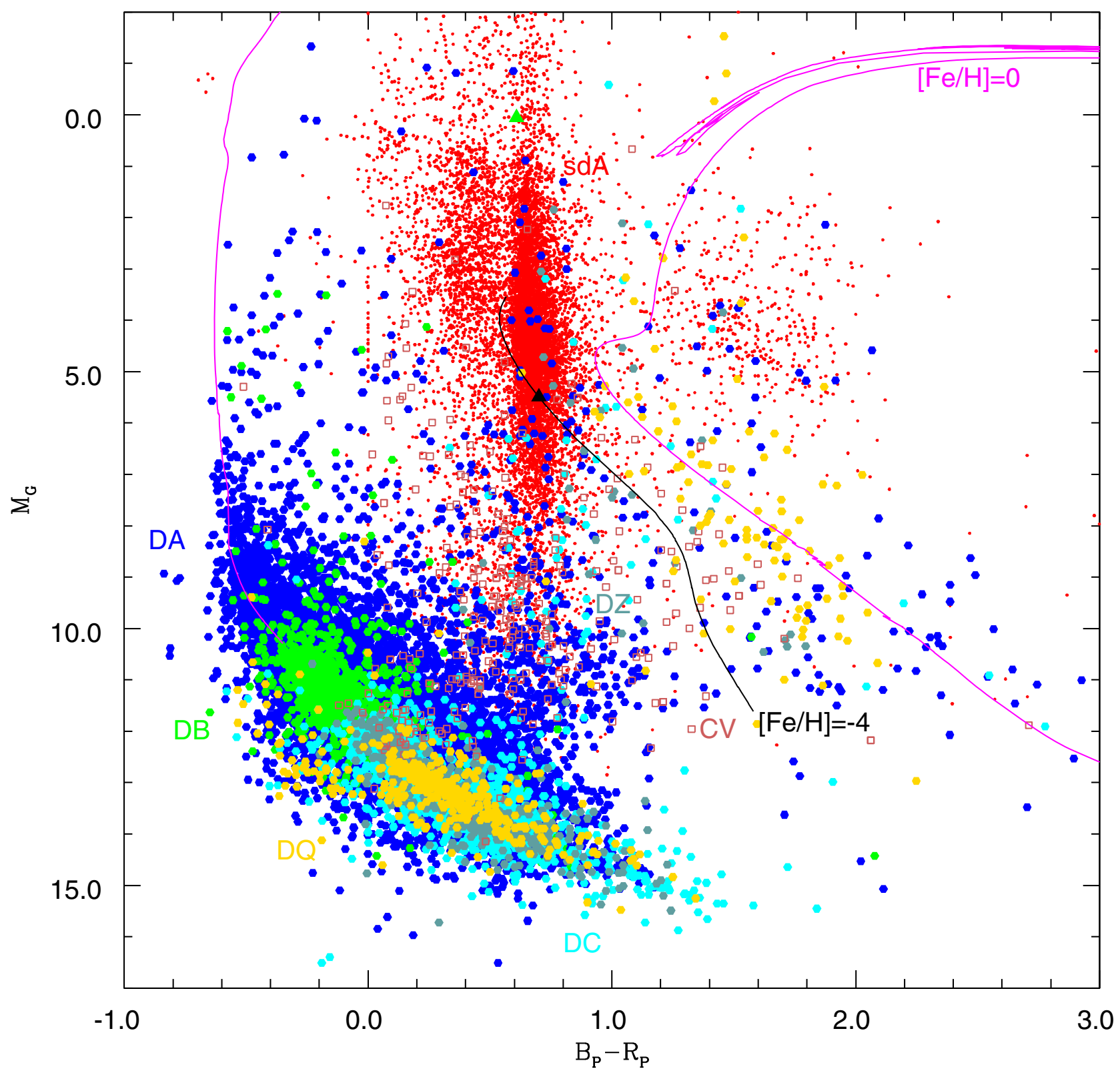

Figure 13. Colour-magnitude diagram of our sample, from Gaia distances and colours. The lines are 10 Gyr MIST isochrones with solar metallicity and $[\mathrm{Fe} / \mathrm{H}]=-4$ (Choi et al. 2018). We also included two triangles, the upper one (green) with $T_{\text {eff }}=6000 \mathrm{~K}, 10 \mathrm{Gyr}$, $[\mathrm{Fe} / \mathrm{H}]=-2.5$, off the main sequence, and the lower one (black), for $[\mathrm{Fe} / \mathrm{H}]=-4$, still on the main sequence, for reference, from the MIST models.

The method is described in Geier et al. (2011). We used appropriate model grids for the different subclasses of sdBs and sdOBs. The hydrogen-rich and helium-poor $[\log y=\log n(\mathrm{He}) / \mathrm{n}(\mathrm{H})<-1.0]$ stars with effective temperatures below $30000 \mathrm{~K}$ were fitted using a of grid of metal line blanketed LTE atmospheres with solar metallicity (Heber et al. 2000). Helium-poor stars with temperatures ranging from 30000 to $40000 \mathrm{~K}$ were analysed using LTE models with enhanced metal line blanketing (O'Toole \& Heber 2006). Metal-free NLTE models (Ströer et al. 2007) were used for hydrogen-rich stars with temperatures below $40000 \mathrm{~K}$ showing moderate He-enrichment $(\log y=-1.0$ to 0.0$)$. The uncertainties provided are from statistical bootstrapping errors only. For more realistic uncertainties, additional random errors of about $\pm 1000 \mathrm{~K}$ in $T_{\text {eff }}$ and $\pm 0.1 \mathrm{dex}$ in $\log g$ should be adopted for $\mathrm{sdBs}$ and
sdOBs. For the hotter sdOs $\pm 2000 \mathrm{~K}$ and $\pm 0.2 \mathrm{dex}$ are more appropriate.

Table 5 shows the hot subdwarfs analysed in this work, their classifications following the scheme proposed in Geier et al. (2017a) and their derived atmospheric parameters from the literature or this work. Fig. 10 shows effective temperature and surface gravity for the sample of hot subdwarf O- and B-type stars.

We also classified 15793 as sdAs, which is only a spectroscopic class to flag objects with narrow $\mathrm{H}$ lines (Kepler et al. 2016). The classification carries no information on their origin or radius. The Gaia DR2 parallax determinations (next section) are somewhat uncertain for these objects (see Fig. 11), being in many cases of the same order of the error. This leads to a large scatter, placing objects above and below the main sequence, the latter a region compatible 
with low-metallicity main-sequence stars, or interacting binary remnants (e.g. Maxted et al. 2014a; Pelisoli et al. 2018a,b, 2019; van Roestel et al. 2018; Wang et al. 2018). Some sdAs could be very low mass white dwarfs or pre-ELMs, but with the current parallax uncertainties this cannot be confirmed. We verified that by simply adding twice the parallax uncertainty to its value, over 95 per cent of the objects in the region between the main sequence and the white dwarf cooling track become compatible with the main sequence, suggesting that an inaccuracy of only $2 \sigma$ in the parallax values is sufficient to explain this spread. Moreover, most of these objects have tangential velocities larger than $200 \mathrm{~km} \mathrm{~s}^{-1}$, the criterion used for halo stars in the Gaia papers, providing further indication that they are compatible with low-mass main-sequence stars in the halo. However, we caution that a high tangential velocity could also be observed for an object in a close binary, which is the case for the ELMs. In the next Gaia data releases, when the astrometry of binary objects and nearby contamination is more accurate, we will have a better understanding of the origin of these sdAs.

Fig. 11 shows the $M_{G}$ versus $G_{\mathrm{BP}}-G_{\mathrm{RP}}$ diagram of the identified sdAs (colour coded by parallax_over_error), with a tentative colour separation for canonical white dwarfs, (pre-)ELM candidates, and stars in the main-sequence region and giant, which might be lowmetallicity main-sequence stars or binaries (e.g. Istrate et al. 2016).

\section{GAIA}

Gaia DR2 listed proper motion for 34499 of our objects, but did not obtain parallax for 4539 of these. The proper motions were mainly compatible with those from the USNO, APOP, and GPS1, and the distances from the parallax are compatible with the spectroscopic distances we obtained. Fig. 12 shows a comparison of the distances estimated from Gaia parallax by Bailer-Jones et al. (2018) versus the distance estimated from our spectroscopic fits for DA stars, showing they are compatible but with a large scatter. The scatter is sometimes caused by the degeneracy of hot and cold solutions in the spectroscopic determination, and low $\mathrm{S} / \mathrm{N}_{g}$, but mainly above magnitude $g=20$ or distances larger than $1.5 \mathrm{kpc}$.

Fig. 13 shows the Hertzprung-Russell colour-magnitude diagram of our DR14 sample, using only the Gaia measurements, totally independent of our spectroscopic measurements. They show DAs and DBs spread through the diagram, compatible with the Kilic et al. (2018) conclusion that the gap seen in Gaia Collaboration (2018) white dwarf HR diagram is not mainly due to atmospheric composition. El-Badry, Rix \& Weisz (2018b), El-Badry et al. (2018a), and El-Badry \& Rix (2018) used the main-sequence white dwarf wide binaries with parallax/error $>20$ for parallax $>10$ mas $(d<100 \mathrm{pc})$ and $M_{G}<14$, corresponding to $T_{\text {eff }}>6000 \mathrm{~K}$, in Gaia DR2, to study the IFMR of white dwarfs, specially for initial masses $<4 \mathrm{M}_{\odot}$, and conclude the bimodality seen in the Gaia data constrains the data to multiple populations.

\section{DISCUSSION}

The systematic uncertainties in our atmospheric parameters derived from spectral analysis are minimized by the use of only SDSS spectra, i.e. same telescope and only two spectrographs (SDSS and BOSS), and fitting all the spectra with the same models and fitting technique.

Gentile Fusillo et al. (2018) selected photometrically white dwarf candidates from the Gaia DR2 and classified those they matched to SDSS spectra in DR14, similar but a subset of our work. We matched their catalogue and we did not miss any star they classified, but we do include other objects they did not classify, because of their selection criteria.

Rolland, Bergeron \& Fontaine (2018) analysed 115 helium-line DBs and 28 cool He-rich hydrogen-line DAs through $\mathrm{S} / \mathrm{N} \geq 50$ spectra and concluded 63 percent of the DBs show hydrogen lines. Koester \& Kepler (2015), using $\mathrm{S} / \mathrm{N}_{g} \simeq 20$ SDSS spectra, measured 75 per cent DBs show hydrogen and speculated all DBs show hydrogen, if observed at high resolution and $\mathrm{S} / \mathrm{N}$. The surface gravity obtained with fits of pure $\mathrm{He}$, for DBs containing $\mathrm{H}$, and of pure $\mathrm{H}$ for DAs containing $\mathrm{He}$, are overestimated, because of the extra particle pressure. Rolland et al. (2018) conclude only if $M_{\mathrm{H}} \geq 10^{-6} M_{*} \mathrm{H}$ will not mix with the underlying He layer by convective mixing at low effective temperatures. In our mean mass determination, we only included DBs hotter than $T_{\text {eff }} \geq 16000 \mathrm{~K}$ and that we did not see any contamination, but it is limited by our $\mathrm{S} / \mathrm{N}$ and resolution. Tremblay et al. (2018) fitted $3171 \mathrm{~S} / \mathrm{N} \geq 20$ DR14 spectra for DAs and 405 DBs for the white dwarfs selected by Gentile Fusillo et al. (2018), applying 3D corrections for both DAs and DBs, as we did, and compared to those they obtain from the Gaia photometry and parallax, concluding the agreement is good, for DAs. They concluded the DA and DB and DBAs mean masses obtained from the Gaia data match within 2 per cent, but their figs 4, 5 , and 12 show the disagreement for DBs, between spectroscopy and Gaia parallaxes is larger when the pure He 3D corrections are applied. As discussed in Section 3.1, the introduction of the pure 3D correction for DBs is the cause of the reduction in the mean mass of DBs, and it is probably not real. Ourique et al. (2019) show there is strong evidence for spectral evolution with effective temperature.

Latour et al. (2018) analysed the hot subdwarfs of the globular cluster $\omega$ Cen, and found a ratio of 26 per cent sdBs $\left(T_{\text {eff }} \leq 30000 \mathrm{~K}\right)$, 10 percent sdOs $\left(T_{\text {eff }} \geq 42,000 \mathrm{~K}\right)$, and the majority as sdBs (intermediate $T_{\text {eff }}$ ). They also found the majority of their sdOBs were helium-enriched, without a counterpart in Galactic field, while we found $33 / 128=26$ per cent of sdOBs are He-sdOBs.

\section{CONCLUSIONS}

We extended our search of white dwarf and subdwarf stars to SDSS DR14. In addition to searching all spectra with significant proper motion for new white dwarfs, we also fitted known DAs and DBs that fell in our selection criteria. The SDSS flux calibration is based on hundreds of comparison stars and in general more accurate than those derived from single night observations. We fitted the spectra of highest $\mathrm{S} / \mathrm{N}$ for each star, taking into account that SDSS re-observes fields and improves the quality of the spectra. Our classifications are independent from previous classifications, and should be considered improvements.

Of the total 37053 objects in our Table 2, only 6 per cent come from plates obtained after DR12, but only 13927 are in the SDSS DR 7 to DR 12 catalogues. The DR7 to DR12 catalogues contain 35590 stars, including 29262 DAs, so our catalogues are not a subset or complete sets, but complementary. The total number of unique stars in Kleinman et al. (2013), Kepler et al. (2015, 2016), and this DR14 catalogue is 52299, with 28681 DAs, 2287 DCs, 2148 DBs, 1126 DZs, 572 DQs, 137 DOs, 4 DS, 396 sdB, 410 sdOs, and $324 \mathrm{CVs}$.

For the first time we include $3 \mathrm{D}$ convection corrections to the derived effective temperatures of DBs, in addition to DAs. The obtained mean masses for DAs and DBs are lower than any previous determinations. For DAs, the main difference was the inclusion of more DAs cooler than $T_{\text {eff }}=10000 \mathrm{~K}$, which show substantially smaller masses, while for DBs the inclusion of the convection 
correction was the main difference. Tremblay et al. (2018) show the disagreement between the spectroscopic determinations and the Gaia parallaxes and colours increases when the 3D correction is applied.

The Gaia distances and colours show there is large spread in the region between cool white dwarfs and cool main-sequence stars. Due to the considerable uncertainty in the parallax (of the same order of the parallax itself for most stars in this region), a reliable separation between different types of sdAs is not possible. This spread causes many stars to be in the region between the main sequence and the white dwarf cooling range, which is compatible with interacting binary evolution. This region is occupied by known sdBs, sdOs, CVs, and WD + MS binaries. The parallax uncertainties suggest most ( $>95$ per cent) of the sdAs in this intermediary reason are consistent with low-mass metal-poor halo stars, but a few could be products of binary evolution such as ELMs.

\section{ACKNOWLEDGEMENTS}

This study was financed in part by the Coordenação de Aperfeiçoamento de Pessoal de Nível Superior - Brasil (CAPES) - Finance Code 001, Conselho Nacional de Desenvolvimento Científico e Tecnológico - Brasil (CNPq), and Fundação de Amparo à Pesquisa do Rio Grande do Sul (FAPERGS) - Brasil. IP acknowledges support from the Deutsche Forschungsgemeinschaft under grant GE2506/12-1. Funding for the Sloan Digital Sky Survey IV has been provided by the Alfred P. Sloan Foundation, the U.S. Department of Energy Office of Science, and the Participating Institutions. SDSS-IV acknowledges support and resources from the Center for High-Performance Computing at the University of Utah. The SDSS web site is www.sdss.org. SDSS-IV is managed by the Astrophysical Research Consortium for the Participating Institutions of the SDSS Collaboration including the Brazilian Participation Group, the Carnegie Institution for Science, Carnegie Mellon University, the Chilean Participation Group, the French Participation Group, Harvard-Smithsonian Center for Astrophysics, Instituto de Astrofísica de Canarias, The Johns Hopkins University, Kavli Institute for the Physics and Mathematics of the Universe (IPMU) / University of Tokyo, Lawrence Berkeley National Laboratory, Leibniz Institut für Astrophysik Potsdam (AIP), MaxPlanck-Institut für Astronomie (MPIA Heidelberg), Max-PlanckInstitut für Astrophysik (MPA Garching), Max-Planck-Institut für Extraterrestrische Physik (MPE), National Astronomical Observatories of China, New Mexico State University, New York University, University of Notre Dame, Observatário Nacional / MCTI, The Ohio State University, Pennsylvania State University, Shanghai Astronomical Observatory, United Kingdom Participation Group, Universidad Nacional Autónoma de México, University of Arizona, University of Colorado Boulder, University of Oxford, University of Portsmouth, University of Utah, University of Virginia, University of Washington, University of Wisconsin, Vanderbilt University, and Yale University.

This research has made use of NASA's Astrophysics Data System Bibliographic Services, SIMBAD data base, operated at CDS, Strasbourg, France, and IRAF, distributed by the National Optical Astronomy Observatory, which is operated by the Association of Universities for Research in Astronomy (AURA) under a cooperative agreement with the National Science Foundation. This work presents results from the European Space Agency (ESA) space mission Gaia. Gaia data are being processed by the Gaia Data Processing and Analysis Consortium (DPAC). Funding for the DPAC is provided by national institutions, in particular the institutions participating in the Gaia MultiLateral Agreement (MLA). The Gaia mission website is https://www.cosmos.esa.int/gaia. The Gaia archive website is https://archives.esac.esa.int/gaia.

The Gaia mission and data processing have financially been supported by, in alphabetical order by country: the Algerian Centre de Recherche en Astronomie, Astrophysique et Géophysique of Bouzareah Observatory; the Austrian Fonds zur Förderung der wissenschaftlichen Forschung (FWF) Hertha Firnberg Programme through grants T359, P20046, and P23737; the BELgian federal Science Policy Office (BELSPO) through various PROgramme de D'eveloppement d'Expériences scientifiques (PRODEX) grants and the Polish Academy of Sciences - Fonds Wetenschappelijk Onderzoek through grant VS.091.16N; the Brazil-France exchange programmes Fundação de Amparo à Pesquisa do Estado de $\mathrm{S}$ ao Paulo (FAPESP) and Coordenação de Aperfeicoamento de Pessoal de Nível Superior (CAPES) - Comité Français d'Evaluation de la Coopération Universitaire et Scientifique avec le Brésil (COFECUB); the Chilean Dirección de Gestión de la Investigación (DGI) at the University of Antofagasta and the Comite Mixto ESOChile; the National Science Foundation of China (NSFC) through grants 11573054 and 11703065; the Czech-Republic Ministry of Education, Youth, and Sports through grant LG 15010, the Czech Space Office through ESA PECS contract 98058, and Charles University Prague through grant PRIMUS/SCI/17; the Danish Ministry of Science; the Estonian Ministry of Education and Research through grant IUT40-1; the European Commission's Sixth Framework Programme through the European Leadership in Space Astrometry (ELSA) Marie Curie Research Training Network (MRTN-CT-2006-033481), through Marie Curie project PIOF-GA2009-255267 (Space AsteroSeismology \& RR Lyrae stars, SASRRL), and through a Marie Curie Transfer-of-Knowledge (ToK) fellowship (MTKD-CT-2004-014188); the European Commission's Seventh Framework Programme through grant FP7-606740 (FP7SPACE-2013-1) for the Gaia European Network for Improved data User Services (GENIUS) and through grant 264895 for the Gaia Research for European Astronomy Training (GREATITN) network; the European Research Council (ERC) through grants 320360 and 647208 and through the European Union's Horizon 2020 research and innovation programme through grants 670519 (Mixing and Angular Momentum tranSport of massIvE stars - MAMSIE) and 687378 (Small Bodies: Near and Far); the European Science Foundation (ESF), in the framework of the Gaia Research for European Astronomy Training Research Network Programme (GREAT-ESF); the European Space Agency (ESA) in the framework of the Gaia project, through the Plan for European Cooperating States (PECS) programme through grants for Slovenia, through contracts C98090 and 4000106398/12/NL/KML for Hungary, and through contract 4000115263/15/NL/IB for Germany; the European Union (EU) through a European Regional Development Fund (ERDF) for Galicia, Spain; the Academy of Finland and the Magnus Ehrnrooth Foundation; the French Centre National de la Recherche Scientifique (CNRS) through action 'Défi MASTODONS' the Centre National d'Etudes Spatiales (CNES), the L'Agence Nationale de la Recherche (ANR) 'Investissements d'avenir' Initiatives D'EXcellence (IDEX) programme Paris Sciences et Lettres (PSL**) through grant ANR-10-IDEX-0001-02, the ANR D'efi de tous les savoirs' (DS10) programme through grant ANR-15-CE31-0007 for project 'Modelling the Milky Way in the Gaia era' (MOD4Gaia), the Région Aquitaine, the Université de Bordeaux, and the Utinam Institute of the Université de FrancheComté, supported by the Région de Franche-Comté and the Institut des Sciences de l'Univers (INSU); the German Aerospace Agency 
(Deutsches Zentrum für Luft- und Raumfahrt e.V., DLR) through grants 50QG0501, 50QG0601, 50QG0602, 50QG0701, 50QG0901, 50QG1001, 50QG1101, 50QG1401, 50QG1402, 50QG1403, and 50QG1404 and the Centre for Information Services and High Performance Computing (ZIH) at the Technische Universität (TU) Dresden for generous allocations of computer time; the Hungarian Academy of Sciences through the Lendület Programme LP201417 and the János Bolyai Research Scholarship (L. Molnár and E. Plachy) and the Hungarian National Research, Development, and Innovation Office through grants NKFIH K-115709, PD116175, and PD-121203; the Science Foundation Ireland (SFI) through a Royal Society - SFI University Research Fellowship (M. Fraser); the Israel Science Foundation (ISF) through grant 848/16; the Agenzia Spaziale Italiana (ASI) through contracts I/037/08/0, I/058/10/0, 2014-025-R.0, and 2014-025-R.1.2015 to the Italian Istituto Nazionale di Astrofisica (INAF), contract 2014049-R.0/1/2 to INAF dedicated to the Space Science Data Centre (SSDC, formerly known as the ASI Sciece Data Centre, ASDC), and contracts I/008/10/0, 2013/030/I.0, 2013-030-I.0.1-2015, and 201617-I.0 to the Aerospace Logistics Technology Engineering Company (ALTEC S.p.A.), and INAF; the Netherlands Organisation for Scientific Research (NWO) through grant NWO-M-614.061.414 and through a VICI grant (A. Helmi) and the Netherlands Research School for Astronomy (NOVA); the Polish National Science Centre through HARMONIA grant 2015/18/M/ST9/00544 and ETIUDA grants 2016/20/S/ST9/00162 and 2016/20/T/ST9/00170; the Portugese Fundação para a Ciência e a Tecnologia (FCT) through grant SFRH/BPD/74697/2010; the Strategic Programmes UID/FIS/00099/2013 for CENTRA and UID/EEA/00066/2013 for UNINOVA; the Slovenian Research Agency through grant P10188; the Spanish Ministry of Economy (MINECO/FEDER, UE) through grants ESP2014-55996-C2-1-R, ESP2014-55996-C2-2-R, ESP2016-80079-C2-1-R, and ESP2016-80079-C2-2-R, the Spanish Ministerio de Economía, Industria y Competitividad through grant AyA2014-55216, the Spanish Ministerio de Educación, Cultura y Deporte (MECD) through grant FPU16/03827, the Institute of Cosmos Sciences University of Barcelona (ICCUB, Unidad de Excelencia 'María de Maeztu') through grant MDM2014-0369, the Xunta de Galicia and the Centros Singulares de Investigación de Galicia for the period 2016-2019 through the Centro de Investigación en Tecnologías de la Información y las Comunicaciones (CITIC), the Red Española de Supercomputación (RES) computer resources at MareNostrum, and the Barcelona Supercomputing Centre - Centro Nacional de Supercomputación (BSC-CNS) through activities AECT-2016-1-0006, AECT-2016-20013, AECT-2016-3-0011, and AECT-2017-1-0020; the Swedish National Space Board (SNSB/Rymdstyrelsen); the Swiss State Secretariat for Education, Research, and Innovation through the ESA PRODEX programme, the Mesures d'Accompagnement, the Swiss Activités Nationales Complémentaires, and the Swiss National Science Foundation; the United Kingdom Rutherford Appleton Laboratory, the United Kingdom Science and Technology Facilities Council (STFC) through grant ST/L006553/1, the United Kingdom Space Agency (UKSA) through grant ST/N000641/1 and ST/N001117/1, as well as a Particle Physics and Astronomy Research Council Grant PP/C503703/1.

\section{REFERENCES}

Abolfathi B. et al., 2018, ApJS, 235, 42

Allende Prieto C. et al., 2008, AJ, 136, 2070
Althaus L. G., Camisassa M. E., Miller Bertolami M. M., Córsico A. H., García-Berro E., 2015, A\&A, 576, A9

Bailer-Jones C. A. L., Rybizki J., Fouesneau M., Mantelet G., Andrae R., 2018, AJ, 156, 58

Barstow M. A., Barstow J. K., Casewell S. L., Holberg J. B., Hubeny I., 2014, MNRAS, 440, 1607

Battich T., Bertolami M. M. M., Córsico A. H., Althaus L. G., 2018, A\&A, 614, A136

Bergeron P., Saffer R. A., Liebert J., 1992, ApJ, 394, 228

Brown W. R., Kilic M., Allende Prieto C., Gianninas A., Kenyon S. J., 2013, ApJ, 769, 66

Brown W. R., Gianninas A., Kilic M., Kenyon S. J., Allende Prieto C., 2016, ApJ, 818, 155

Brown W. R., Kilic M., Gianninas A., 2017, ApJ, 839, 23

Calcaferro L. M., Althaus L. G., Córsico A. H., 2018, A\&A, 614, A49

Choi J., Conroy C., Ting Y.-S., Cargile P. A., Dotter A., Johnson B. D., 2018, ApJ, 863, 65

Córsico A. H., Romero A. D., Althaus L. G., Hermes J. J., 2012, A\&A, 547, A96

Cukanovaite E., Tremblay P.-E., Freytag B., Ludwig H.-G., Bergeron P., 2018, MNRAS, 481, 1522

Doherty C. L., Gil-Pons P., Siess L., Lattanzio J. C., Lau H. H. B., 2015, MNRAS, 446, 2599

Dreizler S., Heber U., Napiwotzki R., Hagen H. J., 1995, A\&A, 303, L53

El-Badry K., Rix H.-W., 2018, MNRAS, 480, 4884

El-Badry K. et al., 2018a, MNRAS, 480, 652

El-Badry K., Rix H.-W., Weisz D. R., 2018b, ApJ, 860, L17

Farihi J., Arendt A. R., Machado H. S., Whitehouse L. J., 2018, MNRAS, 477,3801

Gaia Collaboration, 2018, A\&A, 616, A10

Geier S. et al., 2011, A\&A, 526, A39

Geier S. et al., 2015, A\&A, 577, A26

Geier S., Østensen R. H., Nemeth P., Gentile Fusillo N. P., Gänsicke B. T., Telting J. H., Green E. M., Schaffenroth J., 2017a, A\&A, 600, A50

Geier S. et al., 2017b, Open Astron., 26, 164

Genest-Beaulieu C., Bergeron P., 2019, ApJ, 871, 169

Gentile Fusillo N. P. et al., 2018, MNRAS, 482, 4570

Gianninas A., Dufour P., Kilic M., Brown W. R., Bergeron P., Hermes J. J., 2014, ApJ, 794, 35

Green P., 2013, ApJ, 765, 12

Heber U., 2016, PASP, 128, 082001

Heber U., Reid I. N., Werner K., 2000, A\&A, 363, 198

Ibeling D., Heger A., 2013, ApJ, 765, L43

Istrate A. G., Marchant P., Tauris T. M., Langer N., Stancliffe R. J., Grassitelli L., 2016, A\&A, 595, A35

Kepler S. O. et al., 2015, MNRAS, 446, 4078

Kepler S. O. et al., 2016, MNRAS, 455, 3413

Kilic M., Stanek K. Z., Pinsonneault M. H., 2007, ApJ, 671, 761

Kilic M., Brown W. R., Allende Prieto C., Agüeros M. A., Heinke C., Kenyon S. J., 2011, ApJ, 727, 3

Kilic M., Hambly N. C., Bergeron P., Genest-Beaulieu C., Rowell N., 2018, MNRAS, 479, L113

Kleinman S. J. et al., 2013, ApJS, 204, 5

Koester D., 2010, Mem. Soc. Astron. Ital., 81, 921

Koester D., Kepler S. O., 2015, A\&A, 583, A86

Koester D., Weidemann V., Zeidler E.-M., 1982, A\&A, 116, 147

Koester D., Girven J., Gänsicke B. T., Dufour P., 2011, A\&A, 530, A114

Koester D., Gänsicke B. T., Farihi J., 2014, A\&A, 566, A34

Latour M., Randall S. K., Calamida A., Geier S., Moehler S., 2018, A\&A, 618, A15

Lauffer G. R., Romero A. D., Kepler S. O., 2018, MNRAS, 480, 1547

Lee Y. S. et al., 2008a, AJ, 136, 2050

Lee Y. S. et al., 2008b, AJ, 136, 2022

Li Z., Chen X., Chen H.-L., Han Z., 2019, ApJ, 871, 148

Liebert J., Bergeron P., Holberg J. B., 2005, ApJS, 156, 47

Lindegren L. et al., 2018, A\&A, 616, A2

Margala D., Kirkby D., Dawson K., Bailey S., Blanton M., Schneider D. P., 2016, ApJ, 831, 157 
Marsh T. R., Dhillon V. S., Duck S. R., 1995, MNRAS, 275, 828

Maxted P. F. L. et al., 2014a, MNRAS, 437, 1681

Maxted P. F. L., Serenelli A. M., Marsh T. R., Catalán S., Mahtani D. P., Dhillon V. S., 2014b, MNRAS, 444, 208

Napiwotzki R., 1999, A\&A, 350, 101

O’Toole S. J., Heber U., 2006, A\&A, 452, 579

Ourique G., Romero A. D., Kepler S. O., Koester D., Amaral L. A., 2019, MNRAS, 482, 649

Pelisoli I., Kepler S. O., Koester D., 2018a, MNRAS, 475, 2480

Pelisoli I., Kepler S. O., Koester D., Castanheira B. G., Romero A. D., Fraga L., 2018b, MNRAS, 478, 867

Pelisoli I., Bell K., Kepler S. O., Koester D., Romero A. D., 2019, MNRAS, 482, 3831

Pietrzyński G. et al., 2012, Nature, 484, 75

Ramsay G. et al., 2018, A\&A, 620, A141

Rauch T., Deetjen J. L., 2003, in Hubeny I., Mihalas D., Werner K., eds, ASP Conf. Ser. Vol. 288, Stellar Atmosphere Modeling. Astron. Soc. Pac., San Francisco, p. 103

Reindl N., Rauch T., 2015, in Dufour P., Bergeron P., Fontaine G., eds, ASP Conf. Ser. Vol. 493, TheoSSA - Model WD Spectra on Demand: The Impact of $\mathrm{Ne}, \mathrm{Na}, \mathrm{Mg}$, and Iron-group Elements on the Balmer Line. Astron. Soc. Pac., San Francisco, p. 49

Reindl N., Rauch T., Werner K., Kruk J. W., Todt H., 2014a, A\&A, 566, A116

Reindl N., Rauch T., Werner K., Kepler S. O., Gänsicke B. T., Gentile Fusillo N. P., 2014b, A\&A, 572, 117

Reindl N., Geier S., Kupfer T., Bloemen S., Schaffenroth V., Heber U., Barlow B. N., Østensen R. H., 2016, A\&A, 587, A101

Reindl N., Bainbridge M., Przybilla N, Geier S., Prvák M., Krtička J., Østensen R. H., Telting J., Werner K., 2019, MNRAS, 482, L93

Rivera Sandoval L. E., Maccarone T. J., 2019, MNRAS, 483, A6

Rolland B., Bergeron P., Fontaine G., 2018, ApJ, 857, 56

Roulston B. R., Green P. J., MacLeod C. L., Anderson S. F., Badenes C., 2018, The 20th Cambridge Workshop on Cool Stars, Stellar Systems and the Sun, Boston

Schaeuble M., Falcon R. E., Gomez T. A., Winget D. E., Montgomery M. H., Bailey J. E., 2017, in Tremblay P.-E., Gänsicke B., Marsh T., eds, ASP Conf. Ser. Vol. 509, Helium at White Dwarf Photospheric Conditions: Preliminary Laboratory Results. Astron. Soc. Pac., San Francisco, p. 231

Scholz R.-D., Heber U., Heuser C., Ziegerer E., Geier S., Niederhofer F., 2015, A\&A, 574, A96
Stroeer A., Heber U., Lisker T., Napiwotzki R., Dreizler S., Christlieb N., Reimers D., 2007, A\&A, 462, 269

Sun M., Arras P., 2018, ApJ, 858, 14

Toonen S., Hollands M., Gänsicke B. T., Boekholt T., 2017, A\&A, 602, A16

Tremblay P.-E., Bergeron P., 2009, ApJ, 696, 1755

Tremblay P.-E., Ludwig H.-G., Steffen M., Freytag B., 2013, A\&A, 559, A104

Tremblay P.-E., Kalirai J. S., Soderblom D. R., Cignoni M., Cummings J., 2014, ApJ, 791, 92

Tremblay P.-E., Cukanovaite E., Gentile Fusillo N. P., Cunningham T., Hollands M. A., 2019, MNRAS, 482, 5222

van Roestel J. et al., 2018, MNRAS, 475, 2560

Wang K., Luo C., Zhang X., Zhang B., Deng L., Luo Z., 2018, AJ, 156, 187

Werner K., 1996, ApJ, 457, L39

Werner K., Dreizler S., Heber U., Rauch T., Wisotzki L., Hagen H.-J., 1995, A\&A, 293, L75

Werner K., Deetjen J. L., Dreizler S., Nagel T., Rauch T., Schuh S. L., 2003, in Hubeny I., Mihalas D., Werner K., eds, ASP Conf. Ser. Vol. 288, Stellar Atmosphere Modeling. Astron. Soc. Pac., San Francisco, p. 31

Werner K., Dreizler S., Rauch T., 2012, Astrophysics Source Code Library, record ascl:1212.015

Whitehouse J., Farihi J., Green P. J., Wilson T. G., Subasavage J. P., 2018, MNRAS, 479, 3873

Winget D. E., Hansen C. J., Liebert J., van Horn H. M., Fontaine G., Nather R. E., Kepler S. O., Lamb D. Q., 1987, ApJ, 315, L77

Woosley S. E., Heger A., 2015, ApJ, 810, 34

Zuckerman B., Koester D., Reid I. N., Hünsch M., 2003, ApJ, 596, 477

\section{SUPPORTING INFORMATION}

Supplementary data are available at MNRAS online.

Table 2. Spectral classification.

Table 3. Parameters from spectral fitting to atmospheric models for DAs.

Please note: Oxford University Press is not responsible for the content or functionality of any supporting materials supplied by the authors. Any queries (other than missing material) should be directed to the corresponding author for the article.

This paper has been typeset from a $\mathrm{T}_{\mathrm{E}} \mathrm{X} / \mathrm{L} \mathrm{T} \mathrm{E} \mathrm{X}$ file prepared by the author. 